\title{
DUCTED-FAN ENGINE ACOUSTIC PREDICTIONS USING A NAVIER-STOKES CODE
}

\author{
C. L. Rumsey, R. T. Biedron, F. Farassat \\ Fluid Mechanics and Acoustics Division, NASA Langley Research Center, Hampton, Virginia 23681-2199, USA \\ and P. L. Spence* \\ Lockheed Martin Engineering \& Sciences Co., Hampton, Virginia, USA
}

Published in the Journal of Sound and Vibration

Vol. 213, No. 4, June 1998, pp. 643-664

*currently at Science Applications International Corporation,

Hampton, VA, USA 


\section{Summary}

A Navier-Stokes computer code is used to predict one of the ducted-fan engine acoustic modes that results from rotor-wake/stator-blade interaction. A patched sliding-zone interface is employed to pass information between the moving rotor row and the stationary stator row. The code produces averaged aerodynamic results downstream of the rotor that agree well with a widely used average-passage code. The acoustic mode of interest is generated successfully by the code and is propagated well upstream of the rotor; temporal and spatial numerical resolution are fine enough such that attenuation of the signal is small. Two acoustic codes are used to find the far-field noise. Near-field propagation is computed by using Eversman's wave envelope code, which is based on a finite-element model. Propagation to the far field is accomplished by using the Kirchhoff formula for moving surfaces with the results of the wave envelope code as input data. Comparison of measured and computed far-field noise levels show fair agreement in the range of directivity angles where the peak radiation lobes from the inlet are observed. Although only a single acoustic mode is targeted in this study, the main conclusion is a proof-of-concept: Navier-Stokes codes can be used both to generate and propagate rotor/stator acoustic modes forward through an engine, where the results can be coupled to other farfield noise prediction codes. 


\section{INTRODUCTION}

Ducted-fan engines produce a significant amount of noise that impacts both the communities located near airports as well as the passengers and crew flying in aircraft. Tone noise, which is attributable in large part to the interaction and response of the moving rotor-blade wakes with the stationary stator vanes, is one of the components of engine noise; at the blade passage frequency (BPF) and its higher harmonics, tone noise propagates both forward and aft through the duct and radiates to the far field. Although many methods for analyzing rotor-stator-interaction noise currently exist, most rely extensively on experimental measurements and analytical scaling techniques. As computers continue to become more powerful, however, time-accurate Navier-Stokes computer codes are reaching the point of being able to perform extremely complex three-dimensional (3D), time-accurate acoustic analyses on rotor-stator configurations from first principles, without relying on heuristic techniques.

Theoretical models for rotor-stator interaction acoustic modes have existed for many years, since the first major insight into the problem was given by Tyler and Sofrin [1]. Since that time, the U. S. engine industry has developed several computer codes to model and analyze rotor-stator interaction noise generation. Ventres et al [2] linked two-dimensional (2D) flat-plate cascade theory for unsteady aerodynamics to the 3D annular duct acoustic modes via a strip analysis. Topol [3] later combined this method with a wake model that was originally developed by Majjigi and Gliebe [4]. This empirical wake model was subsequently enhanced to account for more realistic "loaded rotor" type of wakes by Philbrick and Topol [5] and has been combined with other far-field acoustic radiation codes to predict far-field tone noise [6]. Hanson [7] maintains that rotor-stator acoustic interaction theory must account for reflection/transmission effects of the rotor. He developed an analytical theory, based on the linearized unsteady flat-plate cascade method of Smith [8], which includes unsteady coupling between the rotor and the stator, as well as frequency scattering and swirl effects (which can cause "mode trapping"). The effect of finite mean loading is treated by an idealized model via actuator discs that turn the flow at the rotor leading edge and straighten it out at the stator trailing edge.

More advanced computational methods have been applied to rotor-stator flows, but most focus on either global aerodynamic effects or on localized unsteady responses, rather than the generation and propagation of rotor-stator-interaction acoustic modes. Verdon et al [9] solve for the unsteady perturbation field (generally on the stators) that is caused by input waves or blade motions using a linearized, inviscid, unsteady analysis. They have computed unsteady subsonic and transonic flows excited by blade vibrations, acoustic disturbances, and entropic and vortical gusts. Adamczyk et al [10,11] developed an average-passage equation system that governs the time-averaged flow within a typical passage of a blade 
row. They have extensively studied the average aerodynamic properties of multiple blade row systems by using an algebraic turbulence model with wall functions employed to minimize the number of grid points necessary. Several full Navier-Stokes codes have also been applied to rotor-stator-interaction flows [12-18]. In all these studies, the focus of the computations is primarily the prediction of aerodynamic characteristics. Most employ algebraic turbulence models and utilize some type of sliding-zone or clicking-zone interface between the moving rotor and the stationary stator rows.

Several methods have been developed to propagate duct acoustic modes out of the engine to the far field, given that the modes are known a priori. Parrett and Eversman [19] and Roy et al [20] employ a hybrid technique that combines finite elements in the near field with wave-envelope elements in the far field. The axisymmetric formulation is in terms of an acoustic velocity potential. Spence [21] employs a hybrid technique that blends the wave envelope analysis used in Parrett and Eversman [19] with a Kirchhoff integral formula of Farassat and Myers [22] in order to preserve phase information to the far field with minimal storage requirements. Ozyoruk and Long [23] use a high-order spatial scheme with the Euler or Navier-Stokes equations combined with a Kirchhoff surface to propagate the pre-assumed duct acoustic modes forward from the fan face to the far field.

Rangwalla and Rai [24] used the time-accurate thin-layer Navier-Stokes equations to both generate and propagate duct acoustic modes that arise from a 2D rotor-stator interaction. A rescaling strategy was adopted to reduce the number of blades necessary to represent the correct blade-to-vane ratio. They compared the numerically calculated tonal acoustics with theoretical values and found that the predicted acoustics could be affected by the type of numerical boundary conditions employed at the inlet and exit of the computational domain. They also explored the effect of grid spacing and flow nonuniformity on the rate of decay of decaying modes and the ability to maintain propagating modes.

The present work is similar to the approach taken by Rangwalla and Rai, but it pioneers the application of a time-accurate Navier-Stokes code to a realistic 3D configuration. No rescaling of the blades is performed. The present NavierStokes code, which employs upwind differencing and is globally second-order accurate in both space and time, is used to both generate and propagate unsteady duct acoustic modes in a simulation of the 12-inch $(0.3048 \mathrm{~m})$ NASA Langley Research Center advanced ducted propeller (ADP) model [25]. Patched sliding-zone interfaces are employed to pass information between the rotor and stator rows. The effects of spurious reflections from inlet and outlet boundary conditions on the acoustics in the vicinity of the rotor and stator are reduced by extending the computational grid to the far field (outside of the engine) and employing grid stretching there, beyond the region of interest. The Navier-Stokes code is not 
used to propagate the acoustic waves to the far field. Instead, the computed unsteady pressures in the duct forward of the rotor are given as input to a linear wave envelope/Kirchhoff acoustic propagation code [21]. Hence, the Navier-Stokes code needs only enough grid points to adequately resolve and propagate acoustic waves in the near field of the rotor and stator. In the current work, we focus on capturing a particular forward-propagating BPF mode that is "cut on" (propagates) at the test conditions. A previous investigation by Rumsey [26] explored the effect of a sliding-zone interface on the passage of duct acoustic modes. We apply the rule of thumb developed in that reference to ensure that negligible distortion occurs across the sliding-zone interface.

Because this work is one of the first attempts to generate and propagate duct acoustic modes from first principles for a realistic 3D configuration, the primary focus is an analysis of the ability of the Navier-Stokes code to generate a single particular duct acoustic mode and propagate it far enough forward inside the duct to be out of the hydrodynamic field of the rotor. However, a validation exercise is also performed in which the code's global aerodynamic predictive capability is examined, and the far-field noise is compared with measured levels.

\section{PREDICTION CODES}

Three codes are used in the engine noise prediction method reported here. Figure 1 shows the function of each code and the flow of information between the codes. The unsteady aerodynamics code is CFL3D [27,28], developed at NASA Langley Research Center. In the next subsection, the description of this code is given in some detail. The flow field in the interior and exterior regions of the engine is modeled by CFL3D. The numerical computation is run in a time-accurate manner with a high-resolution spatial grid in the vicinity of the rotor blades and the stator vanes to capture the propagating modes of interest. In deciding the spatial and temporal resolution requirements, guidance is sought from wave propagation theory in infinite ducts with uniform flow. As will be shown later, this information is helpful in capturing the propagating modes in the duct. Care is taken to reduce reflections from the outer flow region by stretching the grids away from the high resolution interior region.

To calculate the far-field acoustic pressure, two codes are used as follows. First, a duct propagation and radiation code, based on the finite-element method by Parrett and Eversman [19] and Roy et al [20] (hereafter referred to as Eversman's code), is used to propagate the acoustic modes to the immediate vicinity of the inlet exterior. The information about the acoustic modes is obtained from the CFL3D computation by using a mode decomposition interface. Then, a second acoustic code, based on the Kirchhoff formula for a moving surface, is used to calculate the far-field sound. The input 
data for this code are supplied from near-field data from Eversman's code. The acoustic codes are discussed in subsection 2.2 .

\subsection{DESCRIPTION OF THE UNSTEADY AERODYNAMICS CODE}

The computer code CFL3D solves the 3D time-dependent thin-layer (in each generalized-coordinate direction) Navier-Stokes equations with an upwind finite-volume formulation. The governing equations written in generalized coordinates are

$$
\frac{\partial \hat{Q}}{\partial t}+\frac{\partial\left(\hat{F}-\hat{F}_{v}\right)}{\partial \xi}+\frac{\partial\left(\hat{G}-\hat{G}_{v}\right)}{\partial \eta}+\frac{\partial\left(\hat{H}-\hat{H}_{v}\right)}{\partial \zeta}=0
$$

where

$$
\hat{Q}=\frac{Q}{J}
$$

$Q$ is the vector of conserved variables given as

$$
Q=\left[\begin{array}{c}
\rho \\
\rho u \\
\rho v \\
\rho w \\
e
\end{array}\right]
$$

and $\rho$ is the density, $u, v$, and $w$ are the components of velocity, and $e$ is the total energy per unit volume. The inviscid flux terms are

$$
\begin{gathered}
\hat{F}=\frac{1}{J}\left[\begin{array}{c}
\rho U \\
\rho U u+\xi_{x} p \\
\rho U v+\xi_{y} p \\
\rho U w+\xi_{z} p \\
(e+p) U-\xi_{t} p
\end{array}\right] \\
\hat{G}=\frac{1}{J}\left[\begin{array}{c}
\rho V \\
\rho V u+\eta_{x} p \\
\rho V v+\eta_{y} p \\
\rho V w+\eta_{z} p \\
(e+p) V-\eta_{t} p
\end{array}\right]
\end{gathered}
$$

and 


$$
\hat{H}=\frac{1}{J}\left[\begin{array}{c}
\rho W \\
\rho W u+\zeta_{x} p \\
\rho W v+\zeta_{y} p \\
\rho W w+\zeta_{z} p \\
(e+p) W-\zeta_{t} p
\end{array}\right]
$$

The variable $J$ represents the Jacobian of the transformation:

$$
J=\frac{\partial(\xi, \eta, \zeta)}{\partial(x, y, z)}
$$

The contravariant velocities are given by

$$
\begin{array}{r}
U=\xi_{x} u+\xi_{y} v+\xi_{z} w+\xi_{t} \\
V=\eta_{x} u+\eta_{y} v+\eta_{z} w+\eta_{t}
\end{array}
$$

and

$$
W=\zeta_{x} u+\zeta_{y} v+\zeta_{z} w+\zeta_{t}
$$

The viscous terms are

$$
\begin{gathered}
\hat{F}_{v}=\frac{1}{J}\left[\begin{array}{c}
0 \\
\xi_{x} \tau_{x x}+\xi_{y} \tau_{x y}+\xi_{z} \tau_{x z} \\
\xi_{x} \tau_{y x}+\xi_{y} \tau_{y y}+\xi_{z} \tau_{y z} \\
\xi_{x} \tau_{z x}+\xi_{y} \tau_{z y}+\xi_{z} \tau_{z z} \\
\xi_{x} b_{x}+\xi_{y} b_{y}+\xi_{z} b_{z}
\end{array}\right] \\
\hat{G}_{v}=\frac{1}{J}\left[\begin{array}{c}
0 \\
\eta_{x} \tau_{x x}+\eta_{y} \tau_{x y}+\eta_{z} \tau_{x z} \\
\eta_{x} \tau_{y x}+\eta_{y} \tau_{y y}+\eta_{z} \tau_{y z} \\
\eta_{x} \tau_{z x}+\eta_{y} \tau_{z y}+\eta_{z} \tau_{z z} \\
\eta_{x} b_{x}+\eta_{y} b_{y}+\eta_{z} b_{z}
\end{array}\right]
\end{gathered}
$$

and

$$
\hat{H}_{v}=\frac{1}{J}\left[\begin{array}{c}
0 \\
\zeta_{x} \tau_{x x}+\zeta_{y} \tau_{x y}+\zeta_{z} \tau_{x z} \\
\zeta_{x} \tau_{y x}+\zeta_{y} \tau_{y y}+\zeta_{z} \tau_{y z} \\
\zeta_{x} \tau_{z x}+\zeta_{y} \tau_{z y}+\zeta_{z} \tau_{z z} \\
\zeta_{x} b_{x}+\zeta_{y} b_{y}+\zeta_{z} b_{z}
\end{array}\right]
$$

where 


$$
b_{x_{i}}=u_{j} \tau_{x_{i} x_{j}}-\dot{q}_{x_{i}}
$$

Above,

$$
\tau_{x_{i} x_{j}}=\mu\left(\frac{\partial u_{i}}{\partial x_{j}}+\frac{\partial u_{j}}{\partial x_{i}}\right)-\frac{2 \mu}{3} \frac{\partial u_{k}}{\partial x_{k}} \delta_{i j}
$$

and

$$
\dot{q}_{x_{i}}=-k \frac{\partial T}{\partial x_{i}}
$$

where $\mu$ is the coefficient of molecular viscosity and $k$ is the coefficient of thermal conductivity. The pressure is obtained by the equation of state for a perfect gas:

$$
p=(\gamma-1)\left[e-\rho \frac{\left(u^{2}+v^{2}+w^{2}\right)}{2}\right]
$$

In the CFL3D code, the variables are carried in nondimensional form; they are nondimensionalized by a reference length $L$, a reference density $\rho_{\infty}$, a reference speed of sound $c_{\infty}$, and a reference molecular viscosity $\mu_{\infty}$. For the current application, $L$ is taken as $0.0254 \mathrm{~m}, \rho_{\infty}=1.29 \mathrm{~kg} / \mathrm{m}^{3}, c_{\infty}=340 \mathrm{~m} / \mathrm{s}$, and $\mu_{\infty}=1.8 \times 10^{-5} \mathrm{~kg} /(\mathrm{m}-\mathrm{s})$.

The CFL3D code can solve flows over multiple-zone grids that are connected in a one-to-one, patched, or overset manner. For the current application, nonconservative patching with sliding interfaces as described in Rumsey [26] and Biedron and Thomas [29] is employed between the rotor and stator rows. Upwind-biased spatial differencing with the flux-difference-splitting (FDS) method of Roe [30] is used for the inviscid terms, and flux limiting is used to obtain smooth solutions in the vicinity of shock waves when they occur. Viscous terms are centrally differenced, and a wide variety of both linear and nonlinear turbulence models, including zero-, one-, and two-equation models, are available with the code. The current application employs the eddy-viscosity one-equation field-equation turbulence model of Spalart and Allmaras [31], with the wall function approach of Abdol-Hamid et al [32].

The CFL3D code is advanced implicitly in time with a three-factor approximate factorization (AF) method. The implicit spatial derivatives are first-order accurate, which results in block-tridiagonal inversions for each sweep. However, for solutions that utilize FDS the block-tridiagonal inversions are further simplified with a diagonal algorithm; a spectral radius scaling [33] is employed to account for the effect of the viscous terms. Second-order temporal accuracy for a single-step AF scheme is forfeited for unsteady computations with these simplifications to the left-hand side. Time accuracy 
is of paramount interest here, and two strategies for recovering the desired accuracy through the use of subiterations have been implemented in CFL3D. These strategies were explored in detail by Rumsey et al [27]. The method employed for the computations in this paper is termed the "pseudo time subiteration" (or " $\tau$-TS") method, which uses a second-orderaccurate temporal discretization, as follows.

For a nondeforming mesh, equation (1) can be written as

$$
\frac{1}{J} \frac{\partial Q}{\partial t}=R(Q)
$$

where

$$
R=-\left[\frac{\partial\left(\hat{F}-\hat{F}_{v}\right)}{\partial \xi}+\frac{\partial\left(\hat{G}-\hat{G}_{v}\right)}{\partial \eta}+\frac{\partial\left(\hat{H}-\hat{H}_{v}\right)}{\partial \zeta}\right]
$$

The time term can be discretized with backward differencing:

$$
\frac{(1+\phi)\left(Q^{n+1}-Q^{n}\right)-\phi\left(Q^{n}-Q^{n-1}\right)}{J \Delta t}=R\left(Q^{n+1}\right)
$$

where the superscripts indicate time level. When $\phi=0$, the method is first-order temporally accurate; when $\phi=1 / 2$, the method is second-order accurate. This equation is implicit because the right-hand side is a function of the unknown flow variables at time level $n+1$.

For the $\tau$-TS method in CFL3D, a pseudo time term is added to the time-accurate Navier-Stokes equations:

$$
\begin{gathered}
\frac{\partial Q}{\partial \tau}+\frac{(1+\phi)\left(Q^{n+1}-Q^{n}\right)-\phi\left(Q^{n}-Q^{n-1}\right)}{J \Delta t} \\
=R\left(Q^{n+1}\right)
\end{gathered}
$$

where $\tau$ represents pseudo time. This equation is then discretized and iterated in $m$, where $m$ is the subiteration counter:

$$
\begin{aligned}
& \frac{\left(1+\phi^{\prime}\right)\left(Q^{m+1}-Q^{m}\right)-\phi^{\prime}\left(Q^{m}-Q^{m-1}\right)}{J \Delta \tau}+ \\
& \frac{(1+\phi)\left(Q^{m+1}-Q^{n}\right)-\phi\left(Q^{n}-Q^{n-1}\right)}{J \Delta t}=R\left(Q^{m+1}\right)
\end{aligned}
$$

In equation (22), $\phi$ and $\phi^{\prime}$ govern the order of accuracy of the physical and pseudo time terms, respectively. In practice, the pseudo time term is treated as first order (i.e., $\phi^{\prime}=0$ ), but the general form is shown here for completeness. As $m \rightarrow \infty$, the pseudo time term vanishes and $Q^{m+1} \rightarrow Q^{n+1}$. If $R$ is linearized with 


$$
R\left(Q^{m+1}\right) \cong R\left(Q^{m}\right)+\frac{\partial R}{\partial Q} \Delta Q^{m}
$$

and the quantity $-(1+\phi) Q^{m} /(J \Delta t)$ is added to both sides of equation (22), then equation (22) becomes

$$
\begin{aligned}
& {\left[\left(\frac{1+\phi^{\prime}}{J \Delta \tau}+\frac{1+\phi}{J \Delta t}\right) I+\delta_{\xi} A+\delta_{\eta} B+\delta_{\zeta} C\right] \Delta Q^{m}=} \\
& \frac{\phi^{\prime} \Delta Q^{m-1}}{J \Delta \tau}+\frac{\phi \Delta Q^{n-1}}{J \Delta t}-\frac{(1+\phi)\left(Q^{m}-Q^{n}\right)}{J \Delta t}+R\left(Q^{m}\right)
\end{aligned}
$$

where $\Delta Q^{m}=Q^{m+1}-Q^{m}, A=\partial\left(\hat{F}-\hat{F}_{v}\right) / \partial Q, B=\partial\left(\hat{G}-\hat{G}_{v}\right) / \partial Q$, and $C=\partial\left(\hat{H}-\hat{H}_{v}\right) / \partial Q$. Equation $(24)$ is approximately factored and written in primitive variable form; it is solved as a series of sweeps in each coordinate direction as

$$
\begin{aligned}
& {\left[\left(\frac{\left(1+\phi^{\prime}\right) M}{J \Delta \tau}+\frac{(1+\phi) M}{J \Delta t}\right)+\delta_{\xi} \tilde{A}\right] \Delta q^{\prime}=\frac{\phi^{\prime} M \Delta q^{m-1}}{J \Delta \tau}} \\
& +\frac{\phi M \Delta q^{n-1}}{J \Delta t}-\frac{(1+\phi) M\left(q^{m}-q^{n}\right)}{J \Delta t}+R\left(q^{m}\right) \\
& {\left[\left(\frac{\left(1+\phi^{\prime}\right) M}{J \Delta \tau}+\frac{(1+\phi) M}{J \Delta t}\right)+\delta_{\eta} \tilde{B}\right] \Delta q^{\prime \prime}=} \\
& \left(\frac{\left(1+\phi^{\prime}\right) M}{J \Delta \tau}+\frac{(1+\phi) M}{J \Delta t}\right) \Delta q^{\prime} \\
& {\left[\left(\frac{\left(1+\phi^{\prime}\right) M}{J \Delta \tau}+\frac{(1+\phi) M}{J \Delta t}\right)+\delta_{\zeta} \tilde{C}\right] \Delta q^{m}=} \\
& \left(\frac{\left(1+\phi^{\prime}\right) M}{J \Delta \tau}+\frac{(1+\phi) M}{J \Delta t}\right) \Delta q^{\prime \prime} \\
& q^{m+1}=q^{m}+\Delta q^{m}
\end{aligned}
$$

where the primitive variables are $q=[\rho, u, v, w, p]^{T}, M=\partial Q / \partial q, \tilde{A}=\partial\left(\hat{F}_{-} \hat{F}_{v}\right) / \partial q, \tilde{B}=\partial\left(\hat{G}-\hat{G}_{v}\right) / \partial q$, and $\tilde{C}=\partial\left(\hat{H}-\hat{H}_{v}\right) / \partial q$. The quantity $\Delta \tau$ is based on a constant Courant-Friedrichs-Lewy (CFL) number of 5 . Multigrid technique is used to drive $\Delta q^{m}$ to zero within a reasonable number of subiterations.

Currently, three $\tau$-TS subiterations, in combination with three-level W-type multigrid cycles, are used. This number is sufficient to drive both the mean-flow density residual and the turbulence-model residual down by at least 1 order of magnitude for each iteration for the case considered here.

\subsection{DESCRIPTION OF THE ACOUSTICS CODES}

\subsubsection{Eversman's Duct Propagation and Radiation Code}

Eversman's code is based on the small-perturbation form of the steady-flow potential equation [19]. For the calcula- 
tions reported in this paper, the background flow is assumed to be incompressible and is corrected for compressibility in the duct. A Galerkin finite-element procedure is used to solve the governing wave-propagation equation, which is a linear equation with nonconstant coefficients. In the exterior region of the duct, the Sommerfeld radiation condition is satisfied by using outgoing waves with the method of wave envelope analysis. On the cowl and the hub, the boundary condition for a rigid wall $\partial p^{\prime} / \partial n=0$ is employed when no liner is used, as in the case of the model engine reported here. The variable $p^{\prime}$ is the acoustic pressure.

Eversman's code is applicable for an axially symmetric duct for which the complex amplitude of each mode is specified as a boundary condition at a reference plane where the acoustic computation starts. These modes are obtained from CFL3D pressure results by a Fourier transform in the circumferential direction and a Hankel-like transform in the radial direction. The reference plane must be sufficiently far from the hydrodynamic field of the rotor blades, where large, nonpropagating pressure variations caused by large fluid velocities in the vicinity of the blades may be present. The reference plane can be moved axially as far forward as the start of the hub without substantial changes in the far-field acoustic level, provided that a sufficiently fine grid resolution is maintained in the aerodynamic prediction code. Eversman's code assumes an irrotational mean axial flow at its input plane. Because the Navier-Stokes results are taken well forward of the rotor, the transfer of modal information between the two codes occurs in a region where the irrotational assumption is valid.

In the near-field region of Eversman's code, conventional finite-element grids are used. In general, the cell volumes are taken to be very small in this region; thus, the phase information of the modes is retained. To speed up the computation, large cell volumes are utilized in the intermediate to far-field regions for the wave envelope elements. In general, such large cell volumes work well as a nonreflecting far-field boundary condition. However, in these regions a serious loss of phase information of the acoustic pressure is observed, but the amplitude information is retained. Because recent experimental data from model engines indicate possible interference from the inlet and exhaust radiated noise, the phase of the acoustic pressure needs to be computed as accurately as possible. Sufficient accuracy can be achieved in Eversman's code by reducing the volume of the wave envelope elements. However, this approach substantially increases the computation time, as well as the memory requirements. For this reason, a postprocessor, based on the Kirchhoff formula for moving surfaces, was developed by Spence [21] to take the near field data from Eversman's code and propagate the noise to any distance in the far field. The description of this code is given in the next subsection. 


\subsubsection{Description of the Kirchhoff Postprocessor Code}

This code is based on the Kirchhoff formula for moving surfaces derived by Farassat and Myers [22]. The Kirchhoff surface described by $f(\vec{x}, t)=0$ ( $f>0$ outside the surface) is assumed to be in the linear region of wave propagation, and $p^{\prime}, \partial p^{\prime} / \partial t$, and $\partial p^{\prime} / \partial n$ are specified on this surface. Then, the acoustic pressure in the region outside this surface is given by the following expression:

$$
4 \pi p^{\prime}(x, t)=\int_{S}\left[\frac{E_{1}}{r\left(1-M_{r}\right)}\right]_{r e t} d S+\int_{S}\left[\frac{p^{\prime} E_{2}}{r^{2}\left(1-M_{r}\right)}\right]_{r e t} d S
$$

where $E_{1}$ is a function of the normal, the tangential, and the time derivative of the acoustic pressure, as well as of the kinematic parameters on the Kirchhoff surface. The function $E_{2}$ depends only on the kinematic parameters of this surface. The subscript ret stands for the retarded or the emission time, and $M_{r}$ is the Mach number in the radiation direction, based on the local Mach number of the Kirchhoff surface $S: f=0$. The first term on the right-hand side of equation (29) governs the far field of the acoustic field, whereas the second term governs the near field.

A spherical surface is taken as the Kirchhoff surface in the high-resolution region of the Eversman's code, and the data needed in the Kirchhoff formula are extracted from the computed results. Note that because Eversman's method is in the frequency domain and the Kirchhoff formula is in the time domain, the near-field data of Eversman must be converted to the time domain by multiplying the complex pressure by an appropriate time-dependent spinning function. The Kirchhoff code was developed as a postprocessor for the Eversman's code. The postprocessor was tested by using analytic data on the Kirchhoff surface from a monopole source in motion, and the computed acoustic pressure was in excellent agreement with the analytic result. The details of this code development are documented in Spence [21].

\section{ROTOR-STATOR-INTERACTION INFINITE DUCT THEORY}

This section provides a brief summary of some of the equations that govern the theory of rotor-stator interaction in an infinite duct. Further details can be found in Tyler and Sofrin [1] or Farassat and Myers [34]. Duct acoustic modes are generally characterized as $(m, n)$ modes by their circumferential and radial mode numbers $m$ and $n$. The $m$ modes generated by a rotor-stator interaction are given by

$$
m=h B+k V
$$

where $h$ is the BPF harmonic $(h=1,2,3, \ldots) ; B$ is the number of rotor blades; $V$ is the number of stator vanes; and $k$ is any integer $(\ldots,-2,-1,0,1,2, \ldots)$. For example, for a 16-blade, 20 -vane configuration, the single BPF $(h=1)$ modes 
are: $\ldots,-44,-24,-4,16,36,56, \ldots$ and the $2 \mathrm{BPF}(h=2)$ modes are: $\ldots,-48,-28,-8,12,32,52, \ldots$ Physically, the circumferential mode number indicates the periodicity of the circumferential rotating acoustic mode pattern. A mode of $m=-4$ is periodic over $1 / 4$ of the duct, or $90^{\circ}$, while a mode of $m=16$ is periodic over $1 / 16$ of the duct, or $22.5^{\circ}$. When $m<0$ the pattern rotates in the direction opposite to the rotor rotation.

A solution to the linear wave equation that gives the pressure perturbation levels in an infinite cylindrical duct as a function of radius, circumferential angle, and axial distance is

$$
p(r, \theta, x)=A J_{m}\left(K_{r} r\right) \exp i\left[c K t-m \theta-K_{a} x\right]
$$

where $A$ is the magnitude of the perturbation and $J_{m}$ is the Bessel function of the first kind and order $m$. The frequency is computed by

$$
K=\frac{h B \Omega}{c}
$$

where $\Omega$ is the rotor spin rate and $c$ is the speed of sound. The values of $K_{r}$ are determined from $1 / R$ times the zeros of

$$
J_{m}{ }^{\prime}\left(K_{r} R\right)=0
$$

where $R$ is the radius of the duct. The first zero of (33) represents the first radial order $n=1$, the second zero represents the second radial order $n=2$, and so on. The axial wave number $K_{a}$ is computed from

$$
K_{a}=\frac{K}{\beta^{2}}\left[-M \pm \sqrt{1-\left(\beta K_{r} / K\right)^{2}}\right]
$$

where $\beta=\sqrt{1-M^{2}}$ and $M$ is the Mach number inside the duct; $K_{a}$ is negative for an upstream-moving wave. The spin rate of a given $m$ mode is given by

$$
\Omega_{m}=\frac{h B \Omega}{m}
$$

The cut-off ratio of a particular $(m, n)$ mode is given by

$$
\beta_{m n}=\frac{K}{\beta K_{r}}
$$

If $\beta_{m n}>1$, then the mode propagates; if $\beta_{m n}<1$, then the mode decays. The axial propagation speed of each $m$ mode is given by 


$$
\dot{x}=\frac{h B \Omega}{K_{a}}
$$

and the axial wavelength is given by

$$
\lambda_{a}=\left|\frac{2 \pi}{K_{a}}\right|
$$

The group velocity angle inside the duct, which corresponds to the angle of peak radiation of a particular $(m, n)$ mode, is given for an upstream-moving wave by

$$
\theta_{g}=\operatorname{atan}\left(\frac{K_{r} / K}{\left|K_{a} / K+M / \beta^{2}\right|}\right)=\operatorname{atan}\left(\frac{\beta}{\sqrt{\beta_{m n}^{2}-1}}\right)
$$

As the cut-off ratio $\beta_{m n}$ approaches 1 , the group velocity angle approaches $90^{\circ}$; thus, the energy flux vector is normal to the wall, and the mode does not propagate. The angle of the radiation of a particular mode from the inlet to the far field is given by

$$
\theta_{g}^{\prime}=\operatorname{atan}\left(\frac{\beta_{\infty}}{\left|\left(M_{\infty}-M\right) \beta_{m n}-\sqrt{\beta_{m n}^{2}-1}\right|}\right)
$$

where $M_{\infty}$ is the free-stream Mach number.

In an annular duct, the Bessel function term in (31) is replaced with a sum of the Bessel function of the first kind and a weighted Bessel function of the second kind.

\section{RESULTS}

In Rumsey [35], a 3D model problem was studied in which different duct acoustic modes were specified at a downstream boundary of a cylindrical duct and propagated upstream through a rotating zone. This study, as well as other unpublished work, indicates that patched sliding-zone boundaries pose no fundamental problems for passing acoustic waves. Also, for the second-order-accurate CFL3D code, these studies demonstrate that (a) most modes require on the order of 25 points per wavelength in the axial, circumferential, and radial directions, (b) on the order of 60 time steps per period of oscillation are required, and (c) at least 40 time steps are required for the sliding zone to pass one period of circumferential periodic variation in the flow field. Furthermore, modes with very low cut-off ratios tend to be damped more than modes with higher cut-off ratios.

In the experiment of Thomas et al [25], the ADP model had 16 rotor blades and either 20 or 40 stator vanes; the vanes 
were placed in either a forward or rear position. Various free-stream Mach numbers were tested. We choose to model the configuration with 20 vanes in the forward position, with a free-stream Mach number of 0.2. According to infinite duct linear theory with an estimated internal duct Mach number of 0.6, the only BPF modes that should propagate at this condition are the modes for $m=-4$. For this initial study, we focus on capturing the $(-4,1)$ mode. A grid created with an extension of the TIGER grid-generation code [36] is shown in Figure 2. The grid contains 12 zones with a total of 2.37 million points. The grid covers $90^{\circ}$ (to capture the $(-4,1)$ mode) and includes the external flow field. Four rotor passages and five stator passages are modeled; the four rotor passages rotate at a rate of $16900 \mathrm{rpm}$ (equivalent to a nondimensional rate of $\Omega^{\prime}=0.02102$ revolutions, where $\Omega^{\prime}=\Omega L / c_{\infty}$ and $L$ is the reference length of $0.0254 \mathrm{~m}, c_{\infty}$ is the free-stream speed of sound taken as $340 \mathrm{~m} / \mathrm{s}$, and $\Omega$ is the rotation rate in revolutions per unit time).

Currently, the rotor tip clearance is not modeled. Based on the previous study [35], 41 circumferential points in the rotor zones, 33 circumferential points in the stator zones, and 33 radial points in all internal zones are required to adequately resolve the $(-4,1)$ mode. The axial spacing in the region near the rotors and stators is sufficiently fine to yield at least 20 - 25 points per wavelength for the $(-4,1)$ mode. Additional axial clustering is placed in the regions near the blade and the vane leading and trailing edges. A nondimensional time step (nondimensionalized by $L$ and $c_{\infty}$ ) of 0.05 is employed, which yields 56 time steps per BPF and 45 steps for the sliding zone to pass $90^{\circ}$ of rotating circumferential periodic variation. Note that increasingly higher order circumferential and radial modes, as well as higher harmonics (e.g., 2 BPF tones), are less well resolved than the $(-4,1)$ BPF mode that is the focus of this study.

A side view of part of the ADP geometry is shown in Figure 3. The axial position of zero is the rotor blade stacking axis. We will show aerodynamic predictions between the rotor and stator rows, followed by acoustic predictions both forward of the rotor and between the rotor and stator rows. The CFL3D code is run both on the primary grid (2.37 million points) and on a grid with every other grid point removed ( 0.32 million grid points), until the mass flow reaches a quasisteady-state value. Figures 4 and 5 show averaged total pressure, total temperature, flow angle, and Mach number as a function of fraction span midway between the rotor and stator rows. Results are shown from CFL3D on both the fine grid and the coarse grid; they are compared with results using the average-passage code of Adamczyk et al [10]. These plots show that the averaged aerodynamic results are relatively insensitive to the grid used. The results agree well with the average-passage code results at the same location. Total pressure and temperature data from the experimental results aft of the stators are plotted for qualitative comparison only. Computed mass flow rate for this engine is $9.21 \mathrm{~kg} / \mathrm{s}$, in 
good agreement with the experimentally measured mass flow rate of $9.30 \mathrm{~kg} / \mathrm{s}$.

Figure 6 shows the total nondimensional relative velocity (in the rotating frame) in the rotor wake predicted from the fine- and coarse-grid discretizations as a function of normalized tangential distance for various normalized axial distances behind the rotor trailing edge. These wakes are computed at a radius of approximately $0.127 \mathrm{~m}$ (along the $j=17$ plane on the finest grid). At this radius, the rotor chord $c_{\text {rotor }}$ is approximately $0.0566 \mathrm{~m}$, and the stator leading edge is an axial distance of approximately $x / c_{\text {rotor }}=0.954$ behind the rotor trailing edge. The fine grid yields a slightly deeper and thinner wake at each station.

To analyze the modal content of the acoustic pressure in the duct, a postprocessing code decomposes the pressure field from the CFL3D code at each axial station of interest into its component duct acoustic modes. The result for the $(-4,1)$ mode in the region of the duct forward of the fan face is shown in Figure 7. Note that the direction of flow in this and most figures to follow is right to left, whereas the acoustic waves of interest travel upstream, from left to right. The real and imaginary parts, as well as the magnitude of the mode, are shown. The signal of this mode is seen to be relatively constant in magnitude at an average of roughly 60 - $70 \mathrm{~Pa}$ over the region from the fan face (at an axial distance of approximately $0.02 \mathrm{~m}$ in front of the rotor blade stacking axis) all the way to the hub leading edge at $0.16 \mathrm{~m}$, where the analysis is terminated. The effect of the grid on the computed strength of the $(-4,1)$ mode is shown in Figure 8. Using the grid with 0.32 million points, the magnitude of the mode is significantly diminished to less than $40 \mathrm{~Pa}$ and visibly attenuates with forward distance traveled. Therefore, whereas both the coarse and fine grids are sufficient to adequately resolve the average aerodynamic properties, the coarse grid is clearly not sufficient to capture the $(-4,1)$ acoustic signal. Further computational resources were not available to determine if grid refinement beyond the fine grid would further alter the computed strength of this signal.

The average Mach number in the duct, as a function of axial station in front of the fan, is shown in Figure 9. The average over the entire duct entrance is approximately 0.47 . With this value and a duct radius of $0.1393 \mathrm{~m}$ with infinite duct theory, the theoretical axial wave speed and wavelength for any duct acoustic mode can be determined from equations (37) and (38). This calculation has been done in Table 1 for the $(-4,1),(-4,2)$, and $(-4,3)$ modes, which for this case are the only BPF forward-moving modes that propagate (i.e., $\beta_{m n}>1$ ) according to theory. The cut-off ratios for these modes are 2.61, 1.50, and 1.09, respectively. We can now compare the waves computed from the Navier-Stokes code with the theoretical values to reassure ourselves that we have captured actual duct acoustic modes as opposed to numer- 
ical artifacts. Figure 10 shows the real part of the $(-4,1)$ mode in the duct at two different times. The nondimensional difference in time is 0.75 (nondimensionalized by $L$ and $c_{\infty}$ ). With $c_{\infty}$ taken as $340 \mathrm{~m} / \mathrm{s}$, this time corresponds to approximately $5.6 \times 10^{-5} \mathrm{~s}$. Superimposed on the plot are two distances that correspond to the theoretical wavelength of $\lambda_{\text {theory }}=0.040 \mathrm{~m}$ and to the distance $\Delta x_{\text {theory }}=0.011 \mathrm{~m}$ that the wave should travel in a nondimensional time of 0.75. The agreement between theory and computation is excellent.

Although the grid was originally designed with only the first radial order mode in mind, Figures 11 and 12 include results for the $(-4,2)$ and $(-4,3)$ modes as well. The amplitudes of these modes vary as a function of the axial distance; thus, either the computational grid is insufficiently fine in the radial direction to adequately resolve these modes and/or the variable geometry and duct Mach number have a more significant effect on these modes than on the $(-4,1)$ mode. In spite of the axial variations in amplitude, the computations predict the increasing wave speed and wavelength with increasing radial order as given by the infinite-duct theory.

As mentioned earlier, for this case modes with $m=-4$ are the only forward-moving BPF modes that propagate, according to infinite duct theory. Although the real configuration is by no means an infinite cylindrical duct, for instructional purposes we examine the behavior of some of the other BPF modes as they propagate forward. Figure 13 shows the magnitude of six different modes, plotted on a log scale because of the high levels of the modes with $m=16$ near the fan face. All of the modes decay to below $10^{-4} \mathrm{~Pa}$ in strength prior to reaching the hub leading edge. This degree of decay lends support to the conjecture that the only modes that contribute to the far-field BPF tone noise are those with $m=-4$, which is in agreement with the theory.

It is also instructive to examine the modal decomposition between the rotor and the stator rows. For one thing, we can ascertain whether the sliding-zone interface has any adverse effect on the passage of the acoustic modes; also, the modal magnitudes in this region may yield some insight into the process by which modes are transmitted, reflected, or scattered by their interaction with the moving rotor blades. Note, however, that the modal decomposition used in the analysis assumes Tyler-Sofrin modes, which are formulated for mean axial flow only. The flow field between the rotor and stator rows has significant shear and swirl. Therefore, the modal decomposition in this region is only approximate.

In Figure 14, the magnitude and the real and imaginary parts of the $(-4,1)$ mode are plotted. The interface between the moving rotor row and the stationary stator row is located at an axial distance of $0.0508 \mathrm{~m}$ behind the rotor blade stacking axis. No significant deviation is noted in the wavelike structure or in the magnitude of this mode at or near the inter- 
face, which indicates that this acoustic mode propagates through the interface without noticeable distortion. Although not shown, the $(-4,2)$ and $(-4,3)$ modes also show no noticeable distortion near the interface. The magnitude of the $(-4,1)$ mode within the space between the rotor and stator is relatively constant at a level of almost $300 \mathrm{~Pa}$. Recall that the level of this mode after it passes through the rotor blades is much lower, that is, between 60 - 70 Pa. Therefore, the interaction of this spinning mode with the moving rotor blades results in the loss of nearly 80 percent of its strength. It is possible that the rotor may either reflect or scatter BPF modes like the $(-4,1)$ mode into higher harmonics, as Hanson [7] describes. Unfortunately, because the current grid and time step utilized for this problem are not sufficient to adequately capture harmonics above $1 \mathrm{BPF}$, and because of uncertainties in the applicability of Tyler-Sofrin modal decomposition in this region, further analysis of this type must be deferred to future investigations.

Finally, the modal information for $m=-4$ extracted from the Navier-Stokes code in the duct forward of the fan face is used as input to Eversman's wave-propagation code and a Kirchhoff postprocessor [21]. The resulting sound pressure level (SPL) is compared with the experimental results along a line that passes $1.2192 \mathrm{~m}$ below the centerline of the engine, as indicated by the solid line with open circles shown in Figure 15. Results are plotted as SPL versus microphone angle in Figure 16. To test the sensitivity to the input plane location, two reference planes inside the duct are used as input to compute the far-field noise levels. Some variation is noted in far-field predicted noise at the higher microphone angles; the reference plane located $0.08 \mathrm{~m}$ forward of the rotor blade stacking axis gives higher levels than the reference plane located $0.14 \mathrm{~m}$ forward of the rotor blade stacking axis. This variation is attributed to the fact that the sound directivity at the higher angles is primarily from the $(-4,2)$ and $(-4,3)$ modes, which are not constant through the length of the duct. Therefore the point at which the Navier-Stokes result is extracted is important. On the other hand, the $(-4,1)$ mode has a lower directivity angle and is primarily responsible for the left-hand lobe in the figure at approximately $30^{\circ}$. The strength of this lobe is not significantly altered by the choice of reference plane because the $(-4,1)$ mode has a relatively constant amplitude throughout the duct. The far-field directivity angles of the $(-4,1),(-4,2)$, and $(-4,3)$ modes according to Eversman's code (roughly $30^{\circ}, 55^{\circ}$, and $55^{\circ}$, respectively) do not agree with the results that were obtained by using the theory of equation (40); this theory predicts directivity angles of $17.5^{\mathrm{o}}, 32.8^{\mathrm{o}}$, and $53.4^{\mathrm{o}}$ for the three modes. However, the infinite-duct theoretical results do not take into account the effects of duct geometry or streamline contraction between the free stream and the duct, both of which may affect directivity.

The computed SPLs are within approximately $10 \mathrm{~dB}$ of the experimentally measured levels at the intermediate angles, 
but are considerably lower at both the lowest and highest angles. However, the experimental data at high angles are corrupted by exhaust noise, because no baffles were employed to eliminate the noise. The broadband noise levels measured in the experiment ranged from roughly $73 \mathrm{~dB}$ at $10^{\circ}$ to $84 \mathrm{~dB}$ at $100^{\circ}$. Although broadband source noise is not modeled in the linear acoustic propagation code, these levels alone do not account for the significant underprediction of the computations at $10^{\circ}$. However, preliminary tests in which a circular microphone array was used have shown that BPF noise sources other than the rotor-stator-interaction modes for $m=-4$ are present in the experiment.

The Navier-Stokes computation on the grid with 2.37 million points requires 140 million words of memory (or 59 words per grid point). This computation requires on the order of 2200 iterations to reach periodic quasi-steady state after it has been restarted from a coarser grid solution. On a single processor CRAY C-90, 2200 iterations translates to approximately $78 \mathrm{CPU}$ hours (or $54 \times 10^{-6}$ seconds per grid point per iteration with 3 three-level multigrid subiterations per time step).

\section{CONCLUSIONS}

The rotor-stator-interaction noise in a realistic 3D duct configuration has been computed. A Navier-Stokes computer code that employs a patched sliding-zone interface between the moving rotor grids and the stationary stator grids has been used to both generate and propagate duct acoustic modes within an advanced ducted propeller engine. The code produces averaged aerodynamic results downstream of the rotor that agree well with the results from a widely used average-passage code. The Navier-Stokes code successfully propagates without attenuation a particular blade passage frequency acoustic mode of interest; the space and time steps were designed to capture this mode based on previously determined engineering rules of thumb. The wave speed and wavelength of this and other modes are shown to agree well with infinite-duct theory. The sliding-zone interface has no noticeable adverse effect on the transmission of these waves. The magnitude of the mode of interest is significantly higher before it passes forward through the rotor blades, which suggests that some of its energy may be either reflected or scattered to other modes. The effects of grid size and the sensitivity of the solution to numerical parameters are assessed for both the averaged aerodynamic results and the acoustic predictions.

A linear far-field acoustic propagation code uses the Navier-Stokes unsteady pressures taken at stations forward of the rotor to predict the far-field noise, in fair agreement with experimental results over a narrow range of directivity angles. However, the main conclusion from this study is not so much a specific numerical result as it is a proof-of-concept: Navi- 
er-Stokes codes can be used both to generate and propagate rotor/stator acoustic modes forward through an engine, where the results can be coupled to an acoustic propagation code that predicts noise in the far field. Only a single mode was targeted in this study, although several other modes were also shown for completeness. Computational results that use even finer grids for engine rigs with more exhaustive experimental data (including rotor wake surveys) will be necessary for a more thorough validation of this noise prediction methodology.

In the future, engine noise may be routinely computed from first principles, with little or no reliance on heuristic techniques. Although currently somewhat expensive for use in routine engine design processes, current state-of-the-art Navier-Stokes codes offer the capability to explore the effects of physical processes that may drive some of the highly nonlinear acoustic interactions within engines. By combining the strength of the Navier-Stokes method in the rotor-stator near field with the proven capability of linear acoustic propagation techniques to predict noise in the far field, a powerful analysis tool can be devised to drive future engine designs.

\section{ACKNOWLEDGMENTS}

The authors acknowledge M. Celestina and J. Adamczyk of NASA Lewis Research Center for their assistance in using the average-passage code, as well as R. Thomas of Virginia Polytechnic Institute and State University - Virginia Consortium for Engineering and Science (VCES) for providing the experimental data. Also, the authors acknowledge O. Yamamoto, C. Miller, and D. Huff of NASA Lewis Research Center and M. DeVries of Pratt and Whitney for their help during the initial stages of this work. The computations were performed on the Numerical Aerospace Simulation (NAS) facility CRAY C-90. 


\section{REFERENCES}

1. J. M. TYLER and T. G. SOFRIN 1962 SAE Transactions 70, 309-332. Axial flow compressor noise studies.

2. C. S. VENTRES, M. A. THEOBALD and W. D. MARK 1982 NASA CR-167952. Turbofan noise generation: volume 1: analysis.

3. D. A. TOPOL 1990 AIAA Paper 90-3951. Rotor wake/stator interaction noise - prediction versus data.

4. R. K. MAJJIGI and P. R. GLIEBE 1984 NASA CR-174849 Volume 1. Development of a rotor/wake vortex model. 5. D. A. PHILBRICK and D. A. TOPOL 1993 AIAA Paper 93-4415. Development of a fan noise design system: part 1: system design and source modeling.

6. D. A. TOPOL and D. A. PHILBRICK 1993 Report prepared for NASA Lewis Research Center Under Contract NAS3-25952, Task 10. Fan noise prediction system development: wake model improvements and code evaluations.

7. D. B. HANSON 1994 NASA CR-4506. Coupled two-dimensional cascade theory for noise and unsteady aerodynamics of blade row interactions in turbofans.

8. S. N. SMITH 1973 British Ministry of Defence Report R\&M No. 3709. Discrete frequency sound generation in axial flow turbomachines.

9. J. M. VERDON, M. BARNETT and T. C. AYER 1995 NASA CR-4698. Unsteady aerodynamic models for turbomachinery aeroelastic and aeroacoustic applications.

10. J. J. ADAMCZYK, M. L. CELESTINA, T. A. BEACH and M. BARNETT 1990 ASME Journal of Turbomachinery 112, 370-376. Simulation of three-dimensional viscous flow within a multistage turbine.

11. J. J. ADAMCZYK, M. L. CELESTINA and E. M. GREITZER 1993 ASME Journal of Turbomachinery 115, 2838. The role of tip clearance in high-speed fan stall.

12. M. M. RAI 1989 Journal of Propulsion and Power 5 307-319. Three-dimensional Navier-Stokes simulations of turbine rotor-stator interaction, (two parts).

13. K. L. GUNDY-BURLET, M. M. RAI, and R. P. DRING 1989 AIAA Paper 89-2452. Two-dimensional computations of multi-stage compressor flows using a zonal approach.

14. E. J. HALL and R. A. DELANEY 1993 NASA CR-187126. Investigation of advanced counterrotation blade configuration concepts for high speed turboprop systems.

15. C. L. CHEN and S. R. CHAKRAVARTHY 1990 AIAA Paper 90-1544. Calculation of unsteady rotor/stator interaction. 
16. J. M. JANUS and D. L. WHITFIELD 1990 AIAA Paper 90-0687. Counterrotating prop-fan simulations which feature a relative-motion multiblock grid decomposition enabling arbitrary time steps.

17. A. ARNONE, R. PACCIANI and A. SESTINI 1995 Journal of Fluids Engineering 117, 647-652. Multigrid computations of unsteady rotor-stator interaction using the Navier-Stokes equations.

18. A. A. RANGWALLA and N. K. MADAVAN 1996 AIAA Paper 96-0249. A high-order-accurate Navier-Stokes method for turbomachinery aeroelastic applications.

19. A. V. PARRETT and W. EVERSMAN 1986 AIAA Journal 24, 753-760. Wave envelope and finite element approximations for turbofan noise radiation in flight.

20. I. D. ROY, W. EVERSMAN and H. D. MEYER 1993 Report Prepared for NASA Lewis Research Center Under Contract NAS3-25952, Task 10. Improved finite element modeling of the turbofan engine inlet radiation problem.

21. P. L. SPENCE 1997 AIAA Paper 97-1651. Ducted fan noise prediction using wave envelope analysis and the Kirchhoff formula.

22. F. FARASSAT and M. K. MYERS 1988 Journal of Sound and Vibration 123, 451-460. Extension of Kirchhoff formula to radiation from moving surfaces.

23. Y. OZYORUK and L. N. LONG 1996 AIAA Paper 96-1771. Progress in time-domain calculations of ducted fan noise: multigrid acceleration of a high-resolution CAA scheme.

24. A. A. RANGWALLA and M. M. RAI 1993 Journal of Fluids and Structures 7, 611-637. A numerical analysis of tonal acoustics in rotor-stator interactions.

25. R. H. THOMAS, C. H. GERHOLD, F. FARASSAT, O. L. SANTA MARIA, W. E. NUCKOLLS and D. W. DEVILBISS 1995 AIAA Paper 95-0722. Far field noise of the 12 inch advanced ducted propeller simulator.

26. C. L. RUMSEY 1997 AIAA Journal 35, 263-268. Computation of acoustic waves through sliding-zone interfaces.

27. C. L. RUMSEY, M. D. SANETRIK, R. T. BIEDRON, N. D. MELSON and E. B. PARLETTE 1996 Computers and Fluids 25, 217-236. Efficiency and accuracy of time-accurate turbulent Navier-Stokes computations.

28. J. L. THOMAS, S. L. KRIST and W. K. ANDERSON 1990 AIAA Journal 28, 205-212. Navier-Stokes computations of vortical flows over high aspect-ratio wings.

29. R. T. BIEDRON and J. L. THOMAS 1990 Computing Systems in Engineering, Computational Technology for 
Flight Vehicles 1, 563-576. A generalized patched-grid algorithm with application to the F-18 forebody with actuated control strake.

30. P. L. ROE 1981 Journal of Computational Physics 43, 357-372. Approximate Riemann solvers, parameter vectors, and difference schemes.

31. P. R. SPALART and S. R. ALLMARAS 1994 La Recherche Aerospatiale 1, 5-21. A one-equation turbulence model for aerodynamic flows.

32. K. S. ABDOL-HAMID, B. LAKSHMANAN and J. R. CARLSON 1995 NASA TP-3480. Application of NavierStokes code PAB3D with $k-\varepsilon$ turbulence model to attached and separated flows.

33. T. J. COAKLEY 1983 AIAA Paper 83-1958. Implicit upwind methods for the compressible Navier-Stokes equations.

34. F. FARASSAT and M. K. MYERS 1997 Journal of Sound and Vibration 200, 729-735. A graphical approach to wave propagation in a rigid duct.

35. C. L. RUMSEY 1996 AIAA Paper 96-1752. Computation of acoustic waves through sliding-zone interfaces using an Euler/Navier-Stokes code.

36. M-H. SHIH, B. K. SONI and J. M. JANUS 1994 Mississippi State University publication. TIGER: turbomachinery interactive grid generation / flow simulation system user's manual. 
Rumsey, Christopher L. (NASA Langley)

Table 1. Theoretical wave speed and wavelength in infinite duct with $0.1393 \mathrm{~m}$ radius with Mach number of 0.47 .

\begin{tabular}{ccc}
\hline \hline Mode & Axial wave speed, $\mathrm{m} / \mathrm{s}$ & Axial wavelength, $\mathrm{m}$ \\
\hline$(-4,1)$ & 180 & 0.040 \\
$(-4,2)$ & 207 & 0.046 \\
$(-4,3)$ & 286 & 0.063 \\
\hline \hline
\end{tabular}




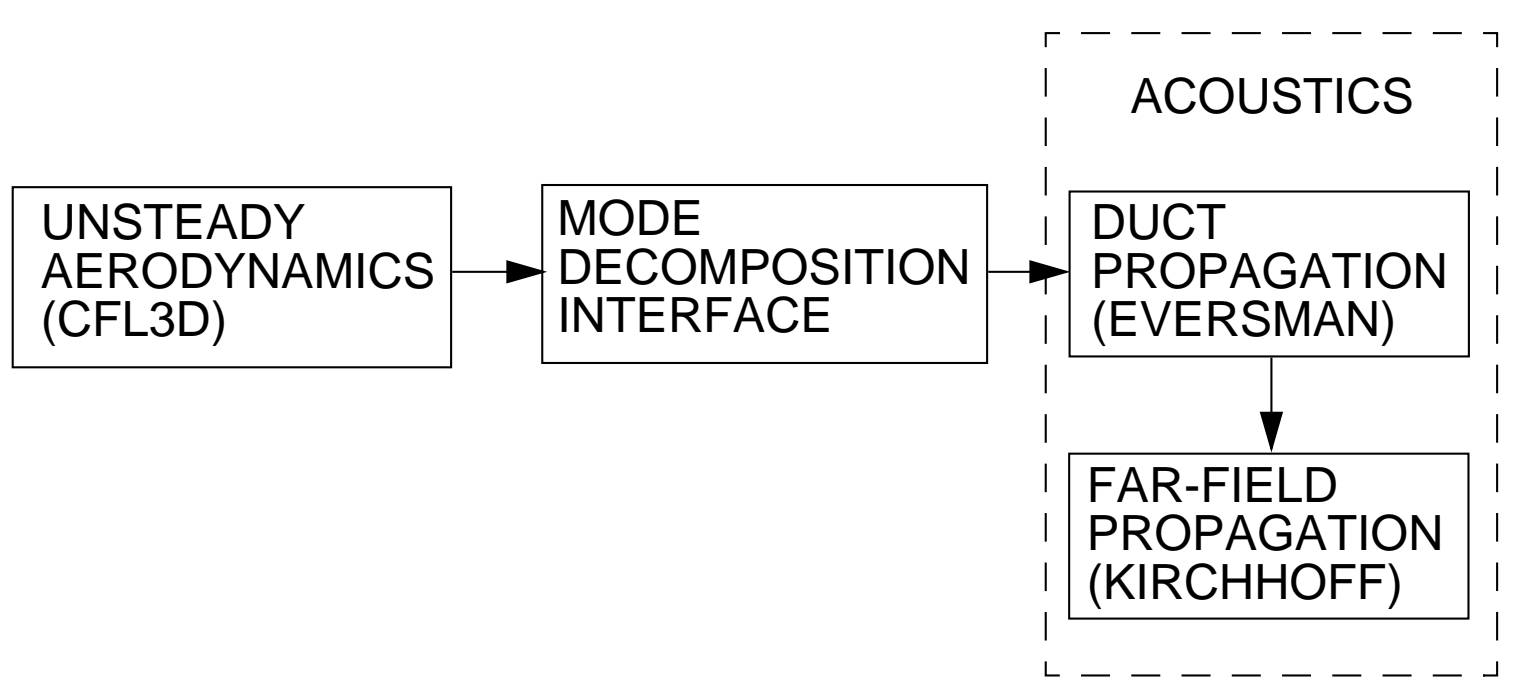

Figure 1. Sketch of ducted-fan engine noise prediction methodology. 


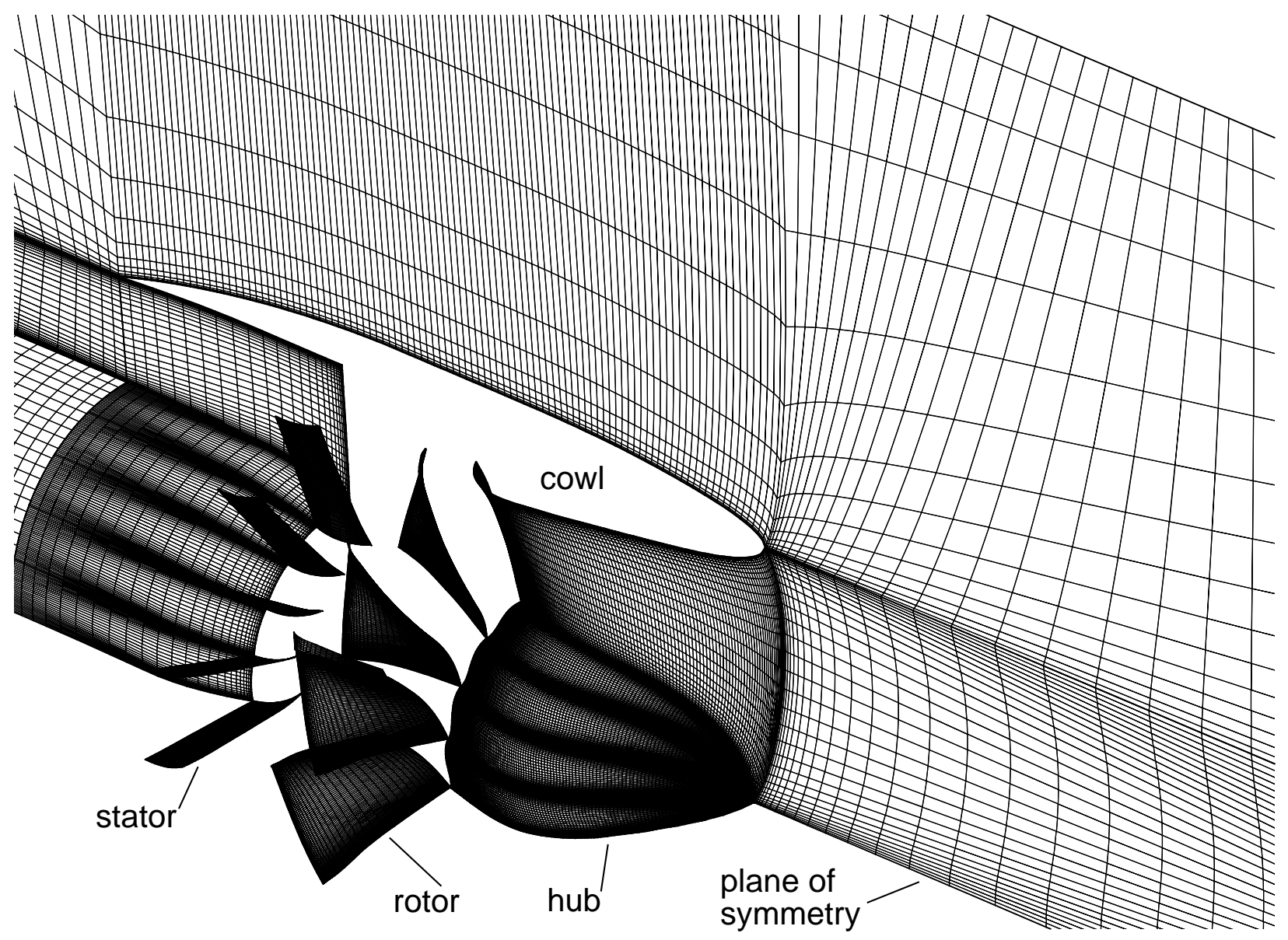

Figure 2. Selected grid cuts from 12-zone grid (2.37 million grid points). 


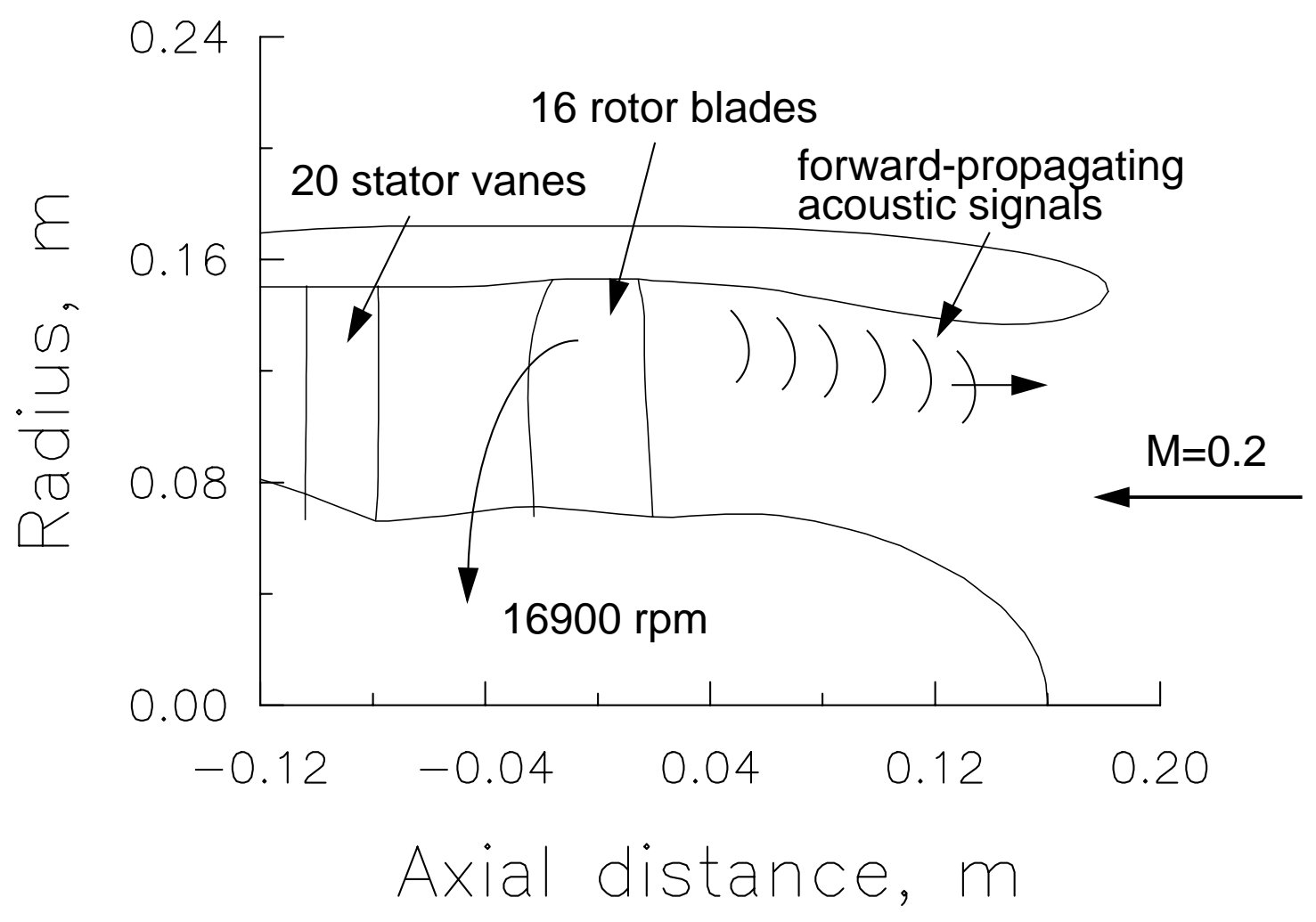

Figure 3. Detail of duct geometry. 

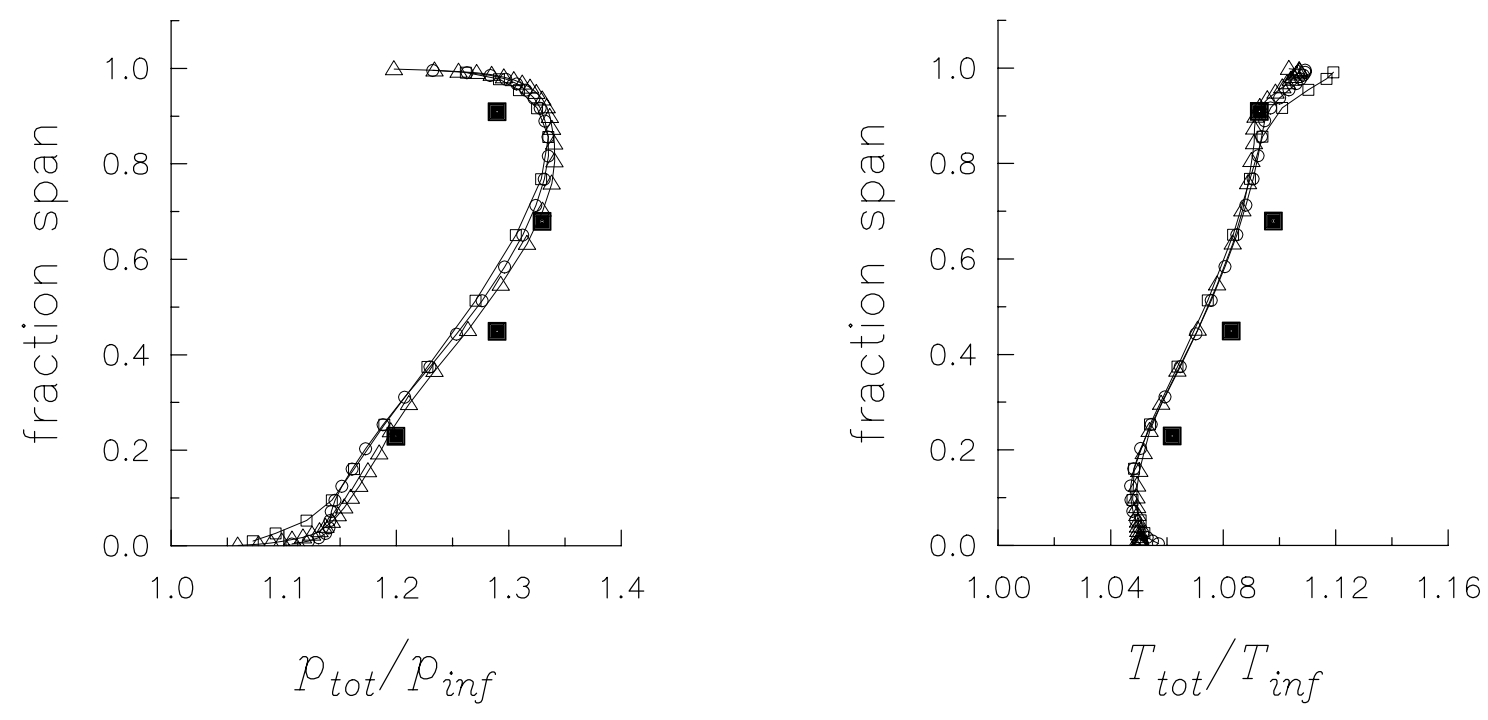

Figure 4. Average results of (a) total pressure and (b) total temperature for $\bigcirc$, CFL3D results behind rotor computed with fine grid; $\square$, CFL3D results behind rotor computed with coarse grid; $\Delta$, Adamczyk average passage code results behind rotor; $\square$, experimental results aft of stator. 

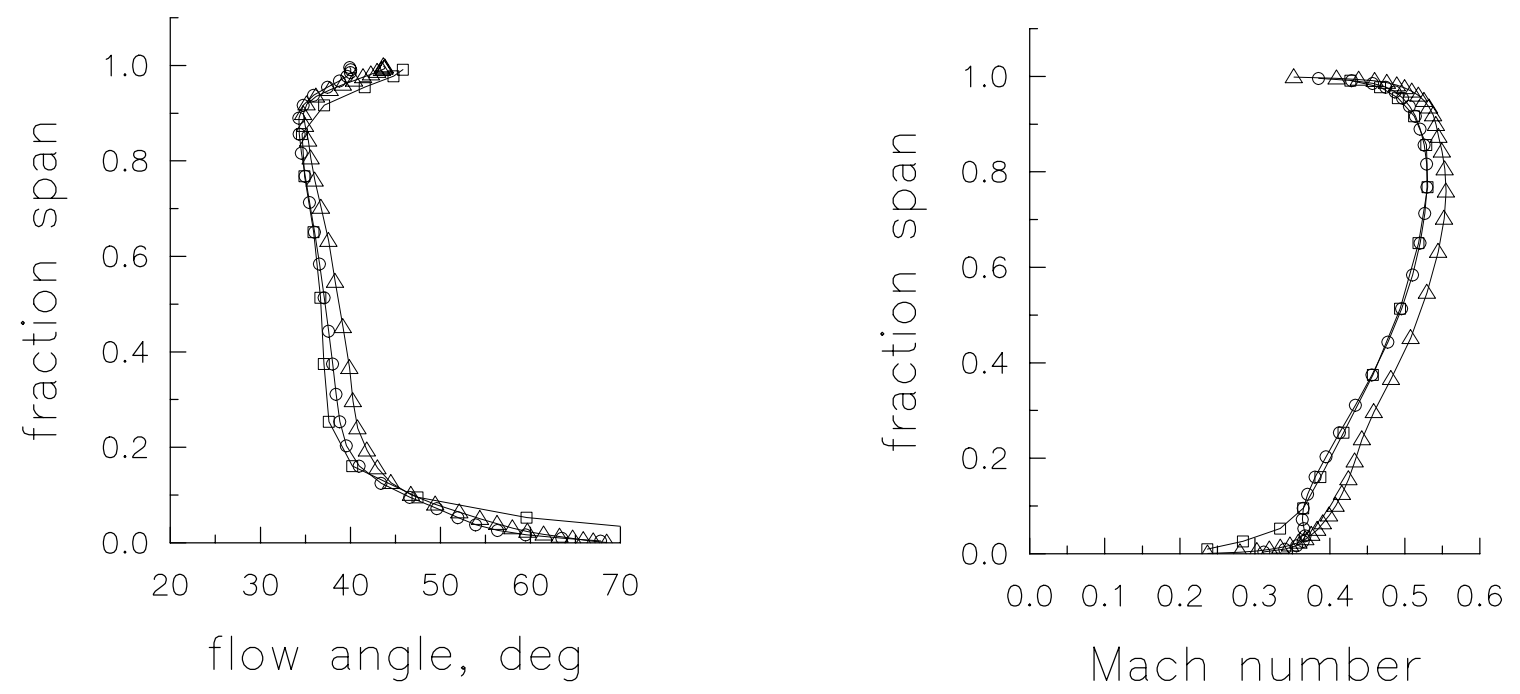

Figure 5. Average results of (a) flow angle and (b) Mach number for $\bigcirc$, CFL3D results behind rotor computed with fine grid; $\square$, CFL3D results behind rotor computed with coarse grid; $\Delta$, Adamczyk average passage code results behind rotor. 


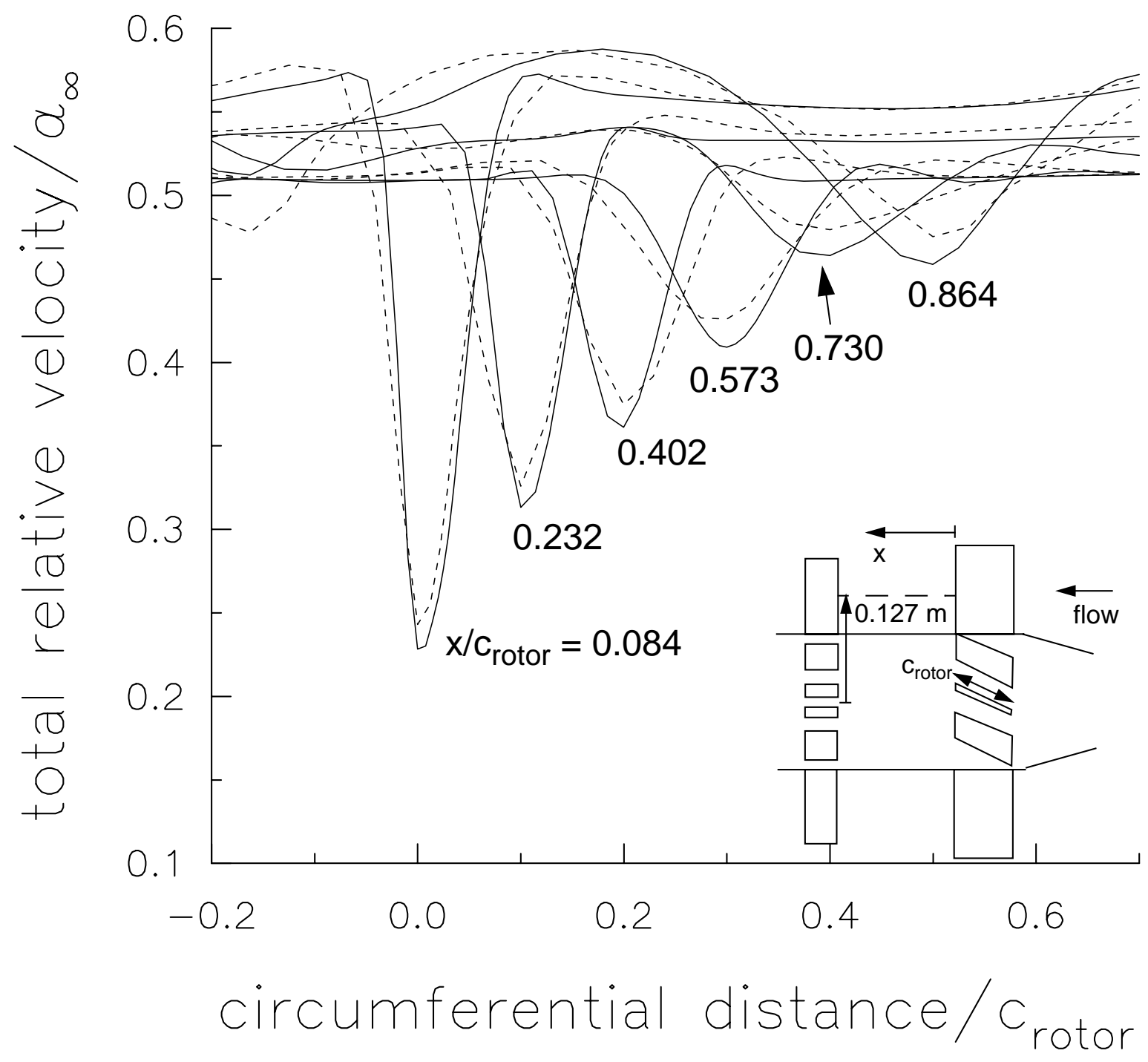

Figure 6. Succession of rotor-wake velocity profiles at various axial distances behind rotor trailing edge at radius of approximately $0.127 \mathrm{~m}$, computed with , fine grid; - . . . . - coarse grid (each successive profile is offset 0.1 units to right on abscissa for clarity). 


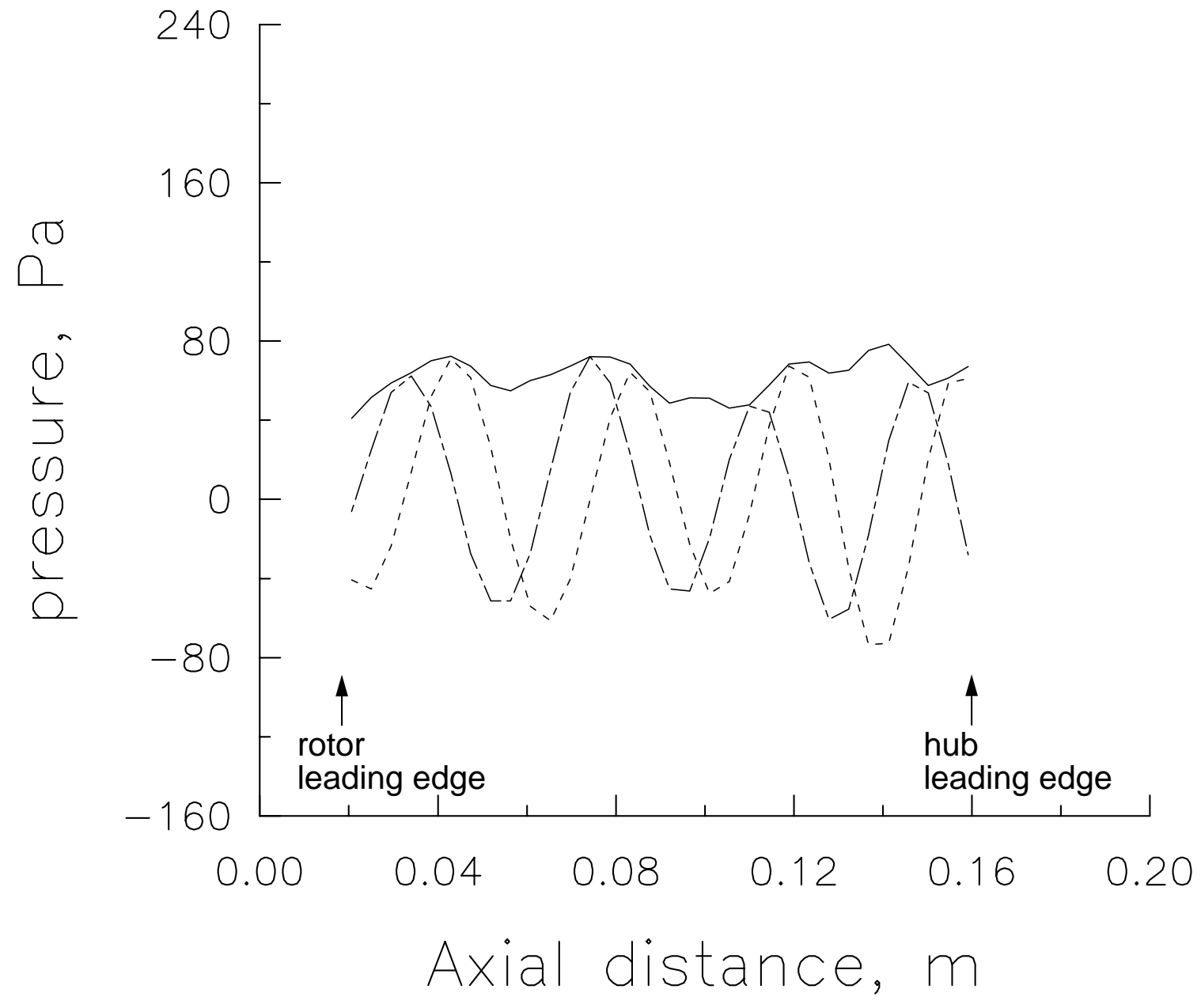

Figure 7. Strength of forward-moving $(-4,1)$ mode in duct between rotor fan face and hub leading edge, computed with fine grid for , magnitude; . . . . . . - , real part; $--\_--$, imaginary part. 


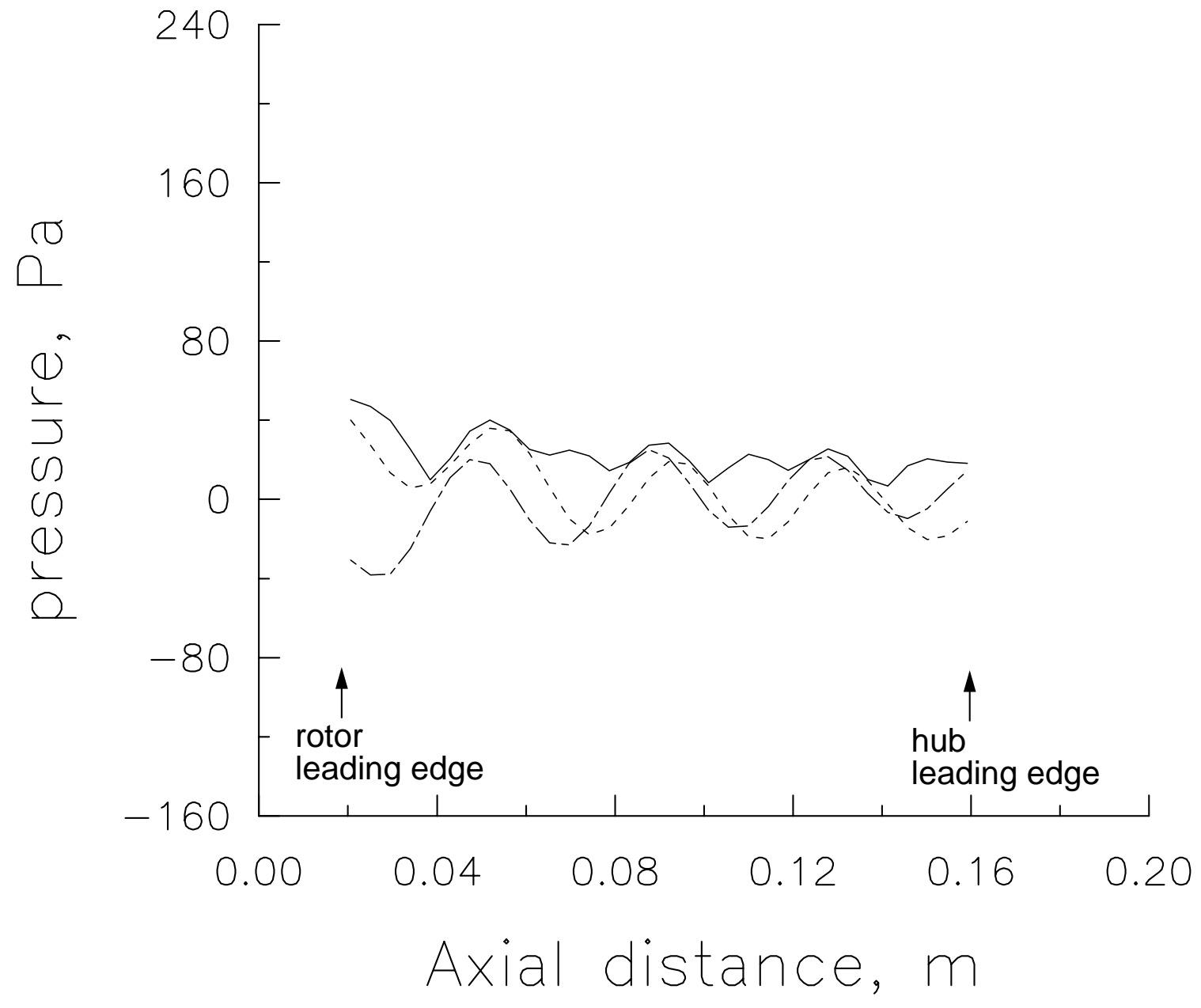

Figure 8. Strength of forward-moving $(-4,1)$ mode in duct between rotor fan face and hub leading edge, computed with coarse grid for $\longrightarrow$, magnitude;.....- -, real part; $-\_---\_$, imaginary part. 


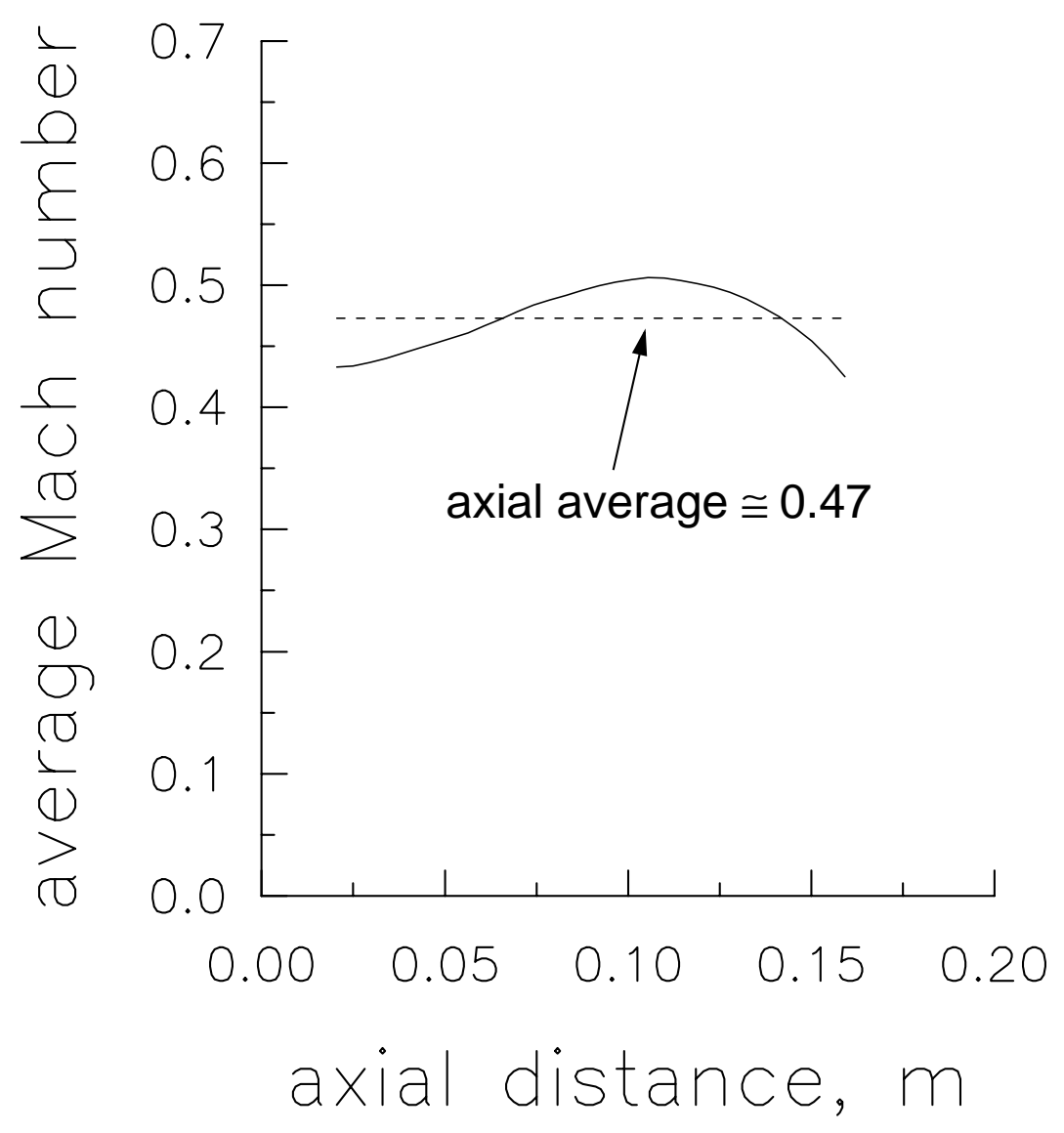

Figure 9. Circumferentially and radially averaged computed Mach number in duct between rotor fan face and hub leading edge. 


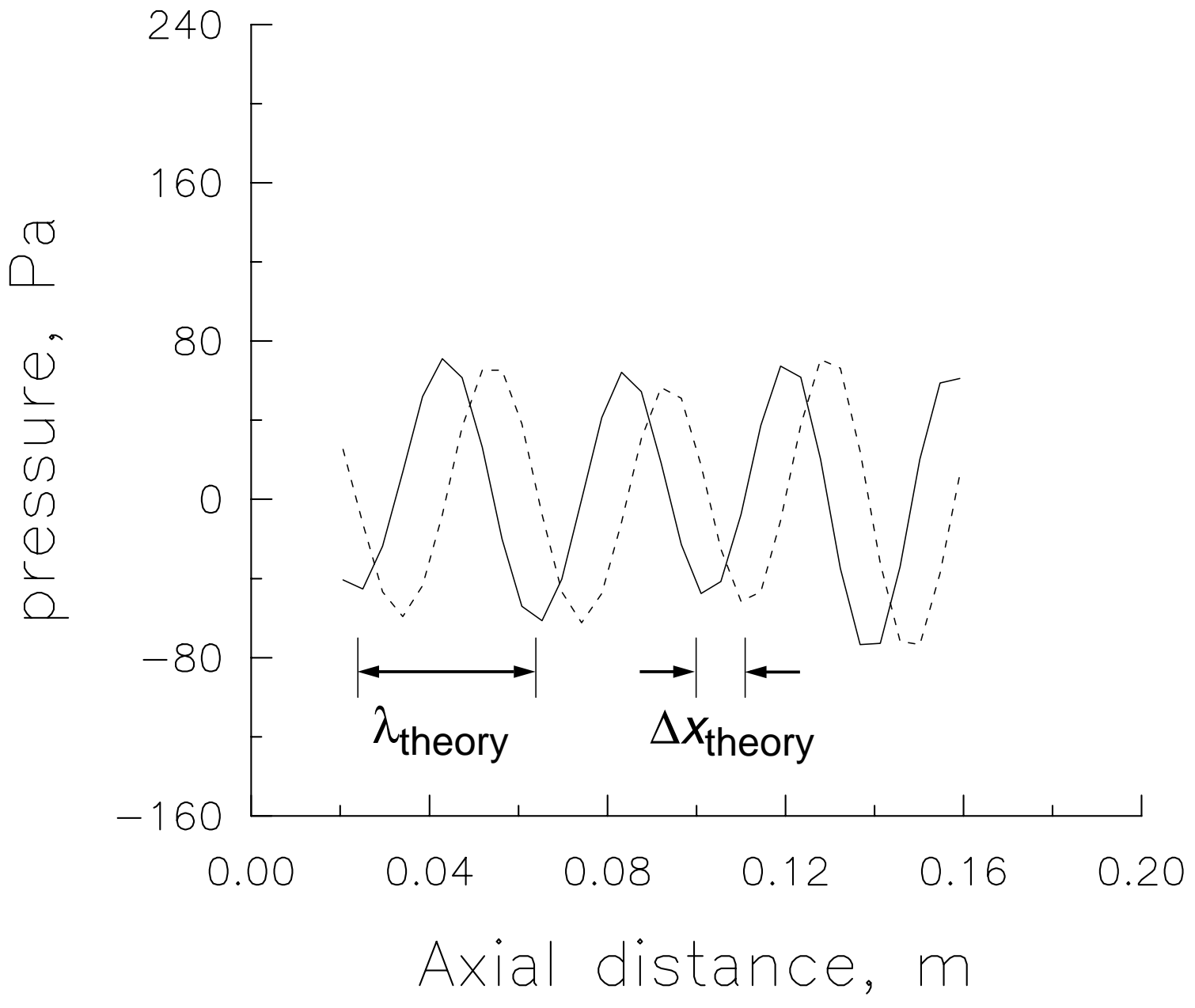

Figure 10. Comparison of computed and theoretical wavelength and wave speed (distance traveled in nondimensional time of 0.75 ) for $(-4,1)$ mode for $\longrightarrow$, time $=\mathbf{T} ; \ldots, \ldots$, time $=\mathbf{T}+\mathbf{0 . 7 5}$. 


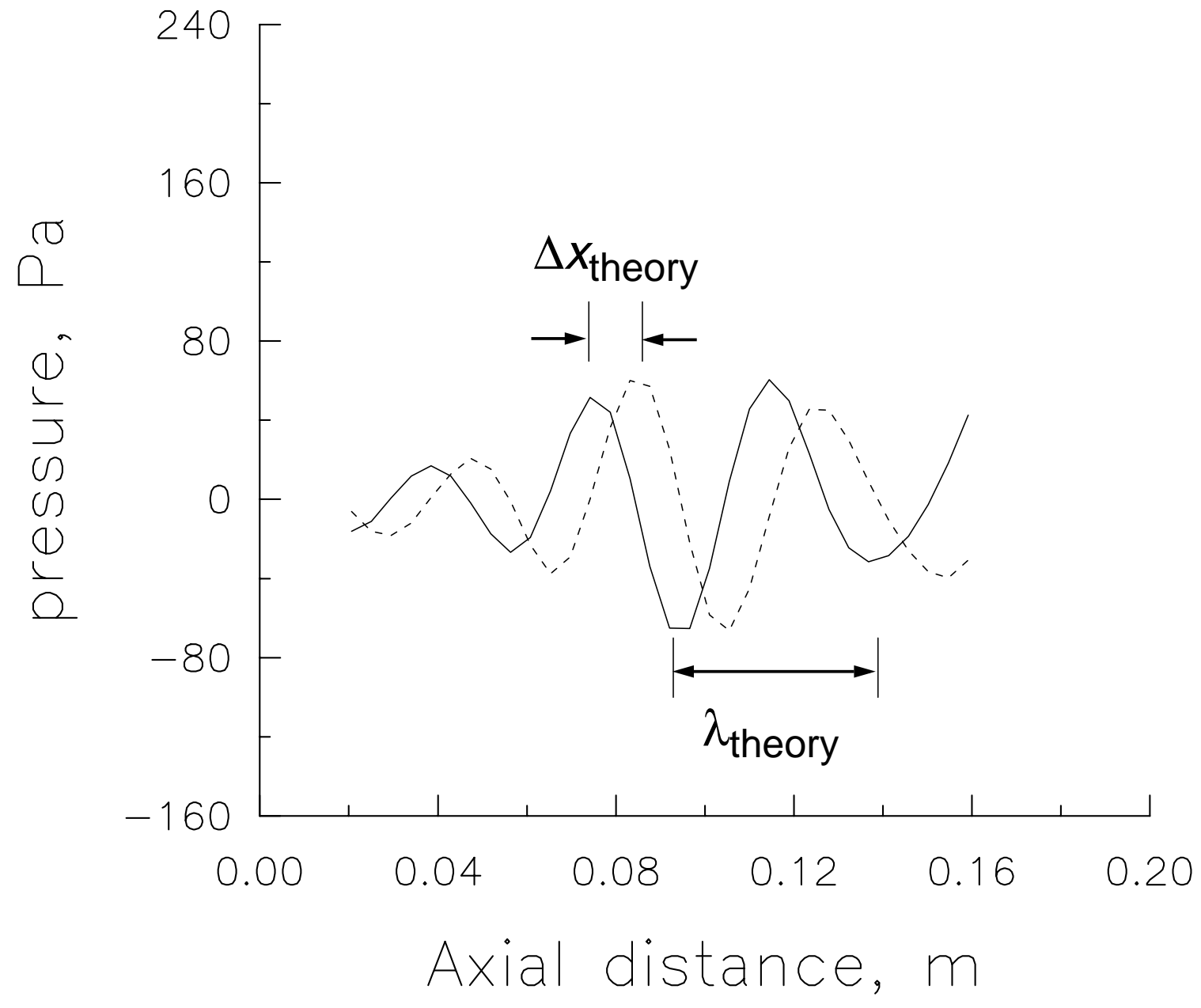

Figure 11. Comparison of computed and theoretical wavelength and wave speed (distance traveled in nondimensional time of 0.75 ) for $(-4,2)$ mode for $\longrightarrow$, time $=\mathbf{T} ; \ldots \ldots,-$, time $=\mathbf{T}+\mathbf{0 . 7 5}$. 


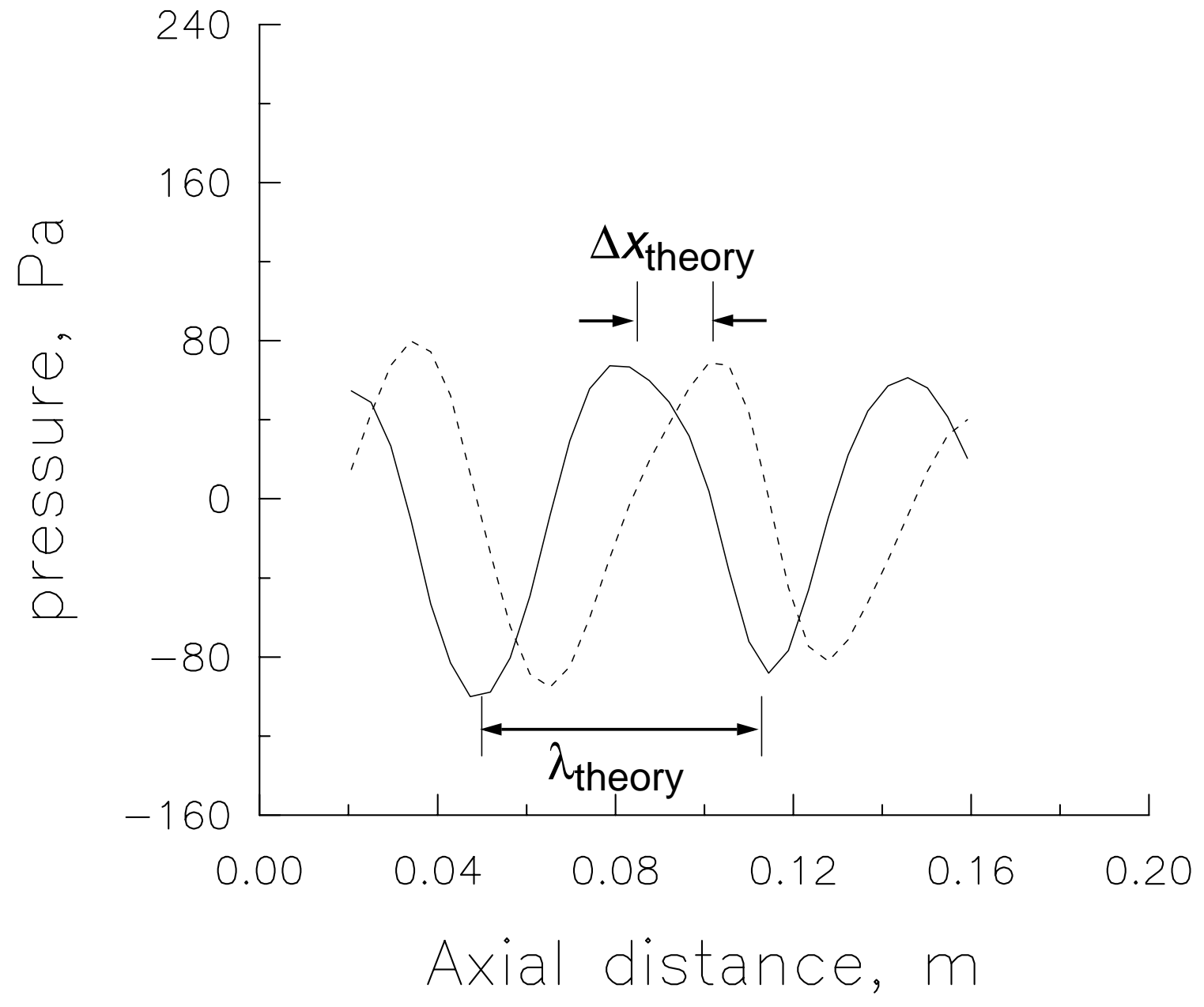

Figure 12. Comparison of computed and theoretical wavelength and wave speed (distance traveled in nondimensional time of 0.75 ) for $(-4,3)$ mode for $\longrightarrow$, time $=\mathbf{T} ; \ldots \ldots$, time $=\mathbf{T}+\mathbf{0 . 7 5}$. 


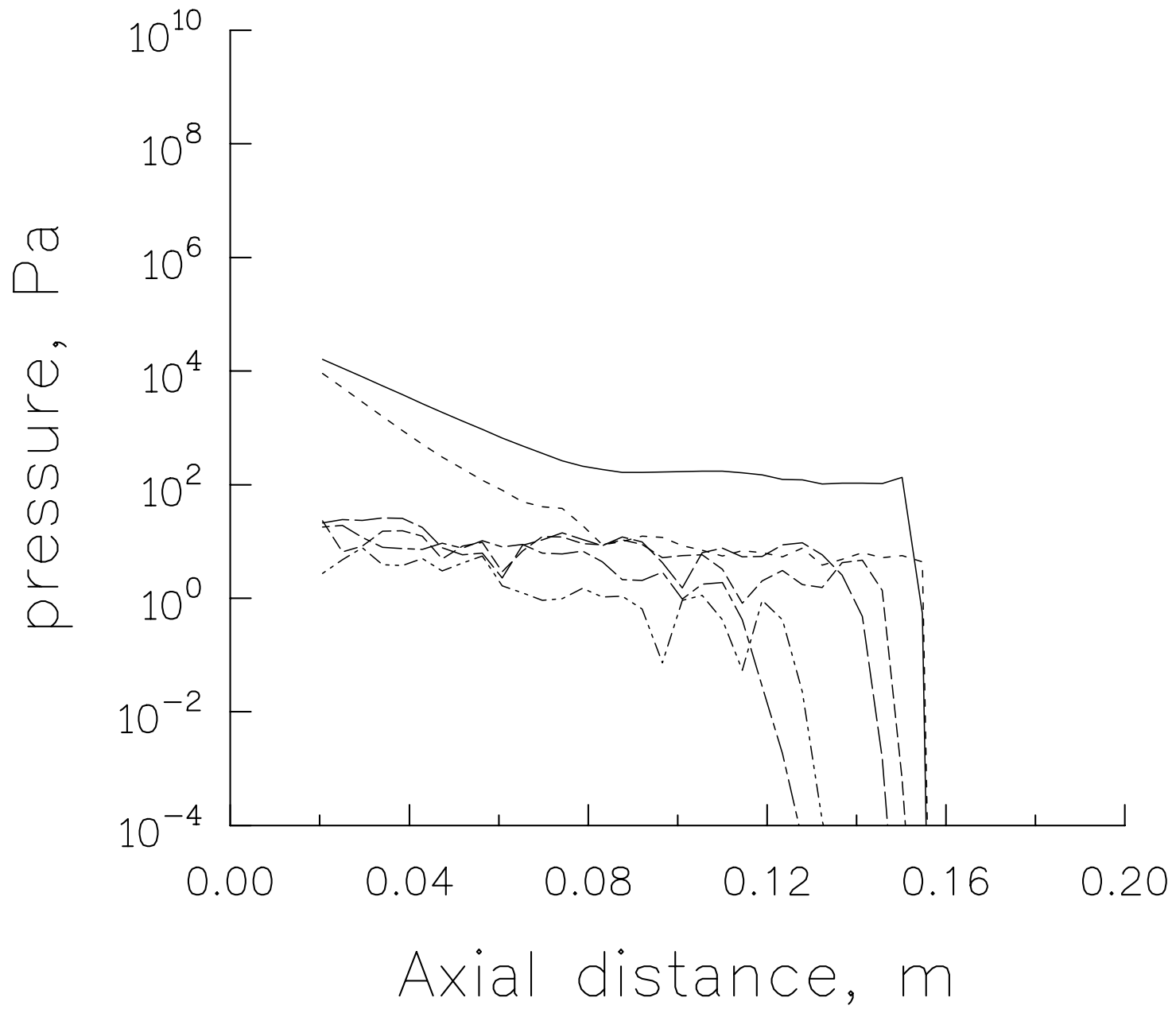

Figure 13. Magnitude of other BPF modes in duct between rotor fan face and hub leading edge: ,$(16,1) ; \ldots \ldots$.

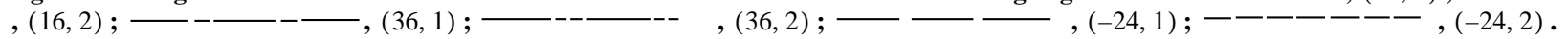




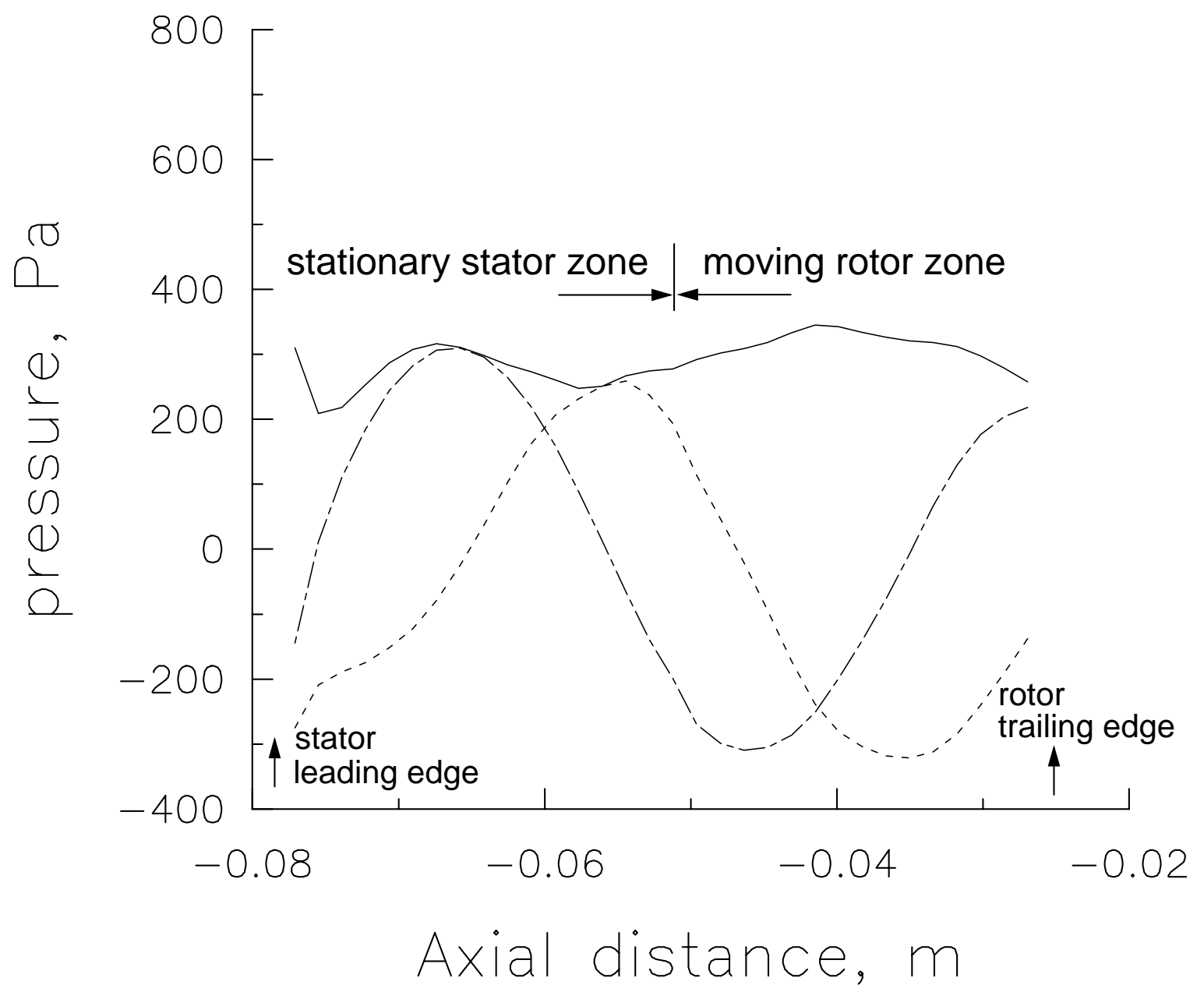

Figure 14. Strength of $(-4,1)$ mode in region between stator leading edge and rotor trailing edge for - , magnitude; - -

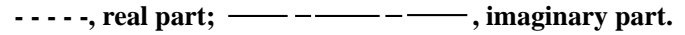




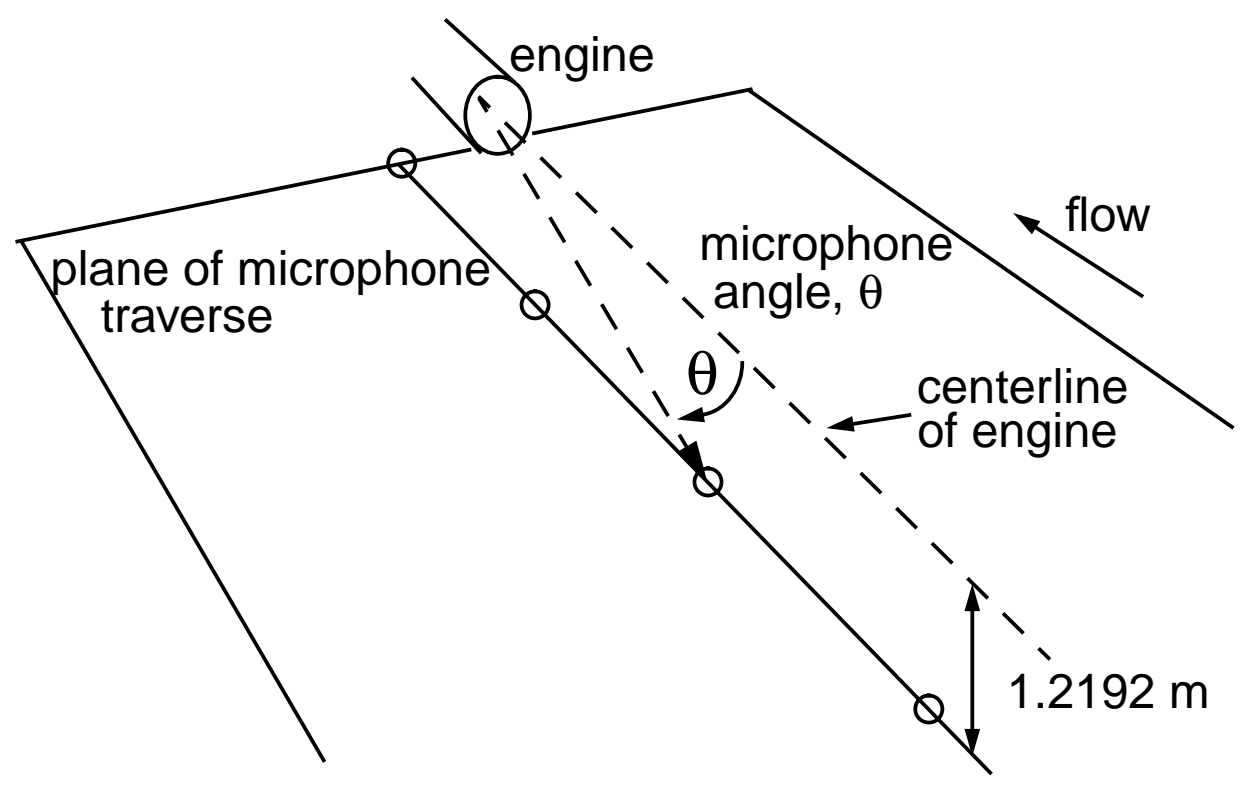

Figure 15. Schematic showing microphone location in experiment of Thomas et al [25]. 


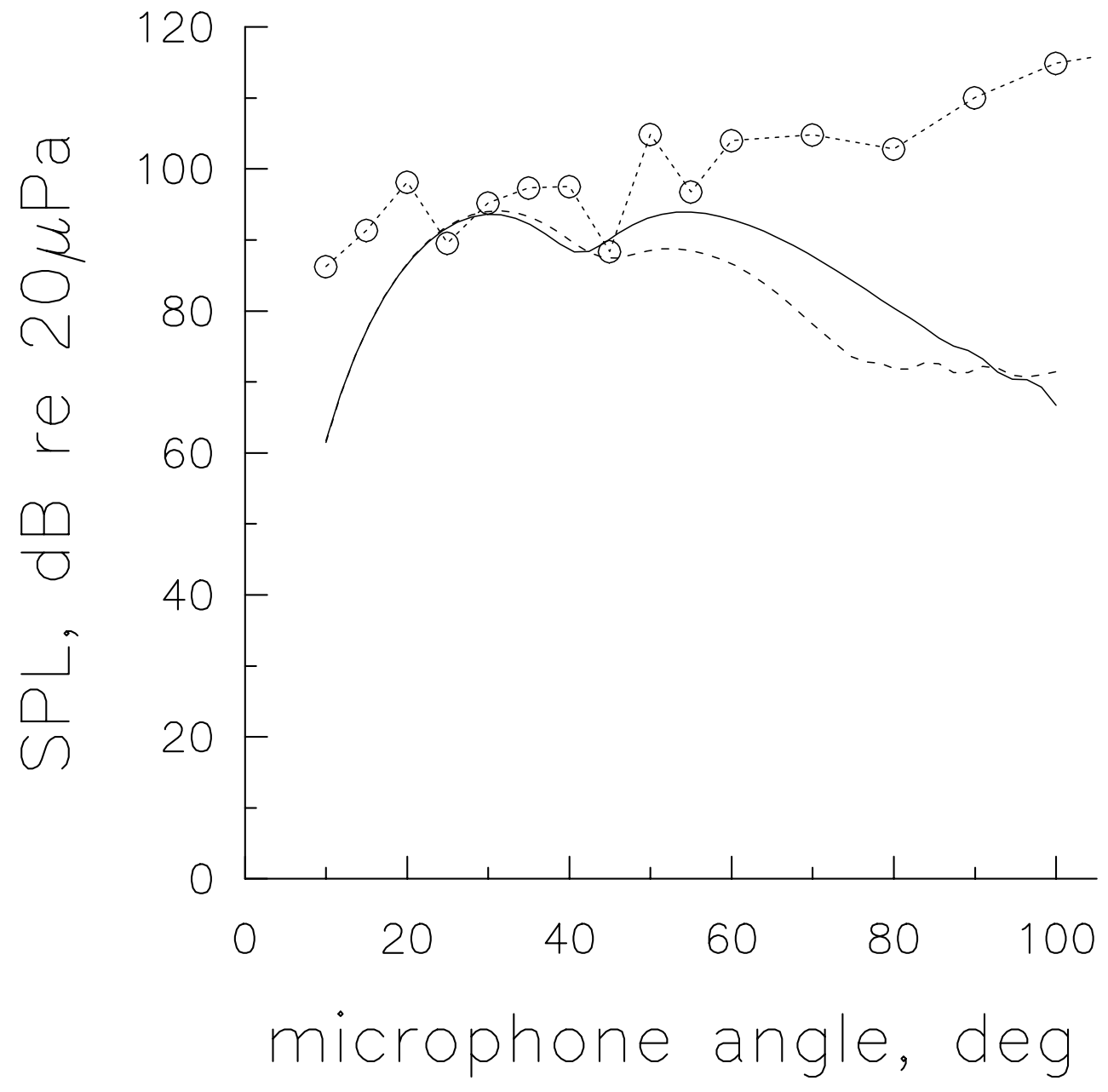

Figure 16. Far-field sound pressure levels:

, using modes with $m=-4$ from Navier-Stokes solution reference plane at $0.08 \mathrm{~m} ;-\ldots-\ldots$, using modes with $m=-4$ from Navier-Stokes solution reference plane at $0.14 \mathrm{~m}$; $\bigcirc$, experimental results. 